\title{
Effect of Inorganic Fertilizer Application on Soil Microbial Diversity in an Oil Palm Plantation
}

Siti Suliza Salamat, ${ }^{\text {a,b }}$ Mohd Ali Hassan, ${ }^{\mathrm{a}, *}$ Yoshihito Shirai, ${ }^{\mathrm{b}}$ Ahmad Husni Mohd. Hanif, ${ }^{\mathrm{c}}$ Mohd Shahkhirat Norizan, ${ }^{\mathrm{d}}$ Mohd Huzairi Mohd Zainudin, ${ }^{\mathrm{e}}$ Nurul Asyifah Mustapha, ${ }^{\mathrm{b}}$ Mohd Noor Mat Isa, ${ }^{\mathrm{f}}$ and Mohd Faizal Abu Bakar ${ }^{\mathrm{f}}$

\begin{abstract}
Excessive fertilizer applications in oil palm plantations are conventionally done to increase the oil yield, but they result in high production cost and environmental pollution. There have been only separate reports on the effects of fertilizer application on soil physical, chemical characteristics, and microbial biodiversity. Therefore, this study was conducted to determine the correlation between soil characteristics and soil microbial biodiversity in oil palm plantation after long-term frequent chemical fertilizer application compared with secondary soil, using molecular methods of polymerase chain reaction denaturing gradient gel electrophoresis (PCR-DGGE) and MiSeq. Secondary forest soil was chosen as the control. The results showed that after 25 years of fertilizer application, the total nitrogen and organic carbon contents decreased from low to very low scale, indicating soil infertility condition. Reduction of Firmicutes was related to suppression of soil borne diseases, and Bacteroidetes which is an indicator of soil health were both almost eliminated after 25 years of fertilizer application. In conclusion, long-term inorganic fertilizer application reduced the soil nitrogen, and organic carbon, altered beneficial microbes in the soil.
\end{abstract}

Keywords: Firmicutes; Bacteroidetes; Inorganic fertilizer application; Oil palm plantation; Soil microbial biodiversity

Contact information: a: Department of Bioprocess Technology, Faculty of Biotechnology and Biomolecular Sciences, Universiti Putra Malaysia, 43400 UPM Serdang, Selangor, Malaysia; b: Department of Biological Functions and Engineering, Graduate School of Life Science and Systems Engineering, Kyushu Institute of Technology, 2-4 Hibikino, Wakamatsu-ku, Kitakyushu, Fukuoka 8080196, Japan; c: Department of Soil Science, Faculty of Agriculture Universiti Putra Malaysia, 43400 UPM Serdang, Selangor, Malaysia; d: Unit Agronomi Sawit, Pusat Penyelidikan Pertanian Tun Razak, FELDA Agricultural Services Sdn Bhd, 26400 Bandar Tun Abdul Razak Jengka, Pahang, Malaysia; e: Institute of Tropical Agriculture and Food Security, Universiti Putra Malaysia, 43400 UPM Serdang, Selangor, Malaysia; f: Malaysian Genome Institute, Jalan Bangi, 43000 Kajang, Selangor, Malaysia;

* Corresponding author: alihas@upm.edu.my

\section{INTRODUCTION}

The second largest area of oil palm plantation in the world is in Malaysia. In 2010 , roughly 5.2 Mha or $16 \%$ of the country's total land area was occupied by oil palm plantation (Gunarso et al. 2013). Almost $60 \%$ of the total forests turned to oil palm plantations were primary forest in Malaysia (Wicke et al. 2011). The limited areas available for oil palm plantation in Malaysia have contributed to the widespread use of a cropping method in the cultivation of palm oil. The impacts of the restricted land region and numerous other influences have induced uninterrupted agriculture such as soil physicochemical degradation and altered microbial profiles. Several of studies have documented the depletion effect of microbial populations. However, the connection between soil productivity and microbial diversity remains uncertain (Berry et al. 2010). Frequently agricultural activities lead to changes on soil properties (top soil and soil compaction) and at the same time reduction in biodiversity (soil microbes). Soil 
productivity and soil microbes are sensitive to the changes in the soil (Agnieszka et al. 2012).

Some studies have shown that the microbial community profile and structure are key determinants of soil health, and these can be affected by various agricultural management practices including frequent fertilizer applications, pesticides, crop rotation, tillage, and use of machinery (Dorr et al. 2012). Long-term continuous cropping contributes to inhibition of plant development and severe soil-borne diseases (Yang et al. 2012; Liu et al. 2014). Various factors have added impediments towards the induction of continuous cropping, including physicochemical degradation of soil characteristics and the build-up of soil-borne pathogens (Fuentes et al. 2009; Huang et al. 2013). Bacteria is the most diverse and abundant class of soil microorganisms that act as indicators of plant health (Gans et al. 2005). Bacteria play key roles as soil-borne pathogens and biocontrol agents. Laborious and costly methods were used previously to determine the dominant soil microbial groups (Roesch et al. 2007). Recently, nextgeneration sequencing (NGS) technology, such as the Illumina Miseq sequencing method, have been used, and these offer an unparalleled potential for fast efficiency and greater visibility in soil bacterial population and profile.

In this study, four replications of soil from an oil palm plantation at the first cycle of a 25-year planting history in the south of Peninsular Malaysia were compared with secondary forest soil within two depth of soil ( 0 to $15 \mathrm{~cm}$ and 15 to $30 \mathrm{~cm})$. The effects of inorganic fertilizer used for continuous-cropping on soil physiochemical characteristics and soil microbial diversity were studied. The objectives were to study the correlation between soil physiochemical properties and soil health to elucidate changes in soil bacterial communities of oil palm plantation under the continuouscropping system for one plantation cycle.

\section{EXPERIMENTAL}

\section{Materials}

The soil samples were collected (Fig. 1) for field sites of oil palm plantation and secondary forest at Serting Hilir Negeri Sembilan. Field sites were situated in the enrichment planting area (Latitude $2.935113^{\circ}$, Longitude $102.464371^{\circ}$ ). The soil series was named as Kechor Soil Series and categorized under Typic Kandiudults according to Paramananthan (2020). This soil was developed over non-accreting sub-recent alluvium (T2) with fine, kaolinitic, isohyperthermic, and yellow in colour. It has not more than $30 \%$ silt content, and the drainage class was categorised as well draining. Generally, the cation exchange capacity (CEC) was not more than $16 \mathrm{cmol}(+) \mathrm{kg}^{-1}$ clay. Clay decreased by more than $20 \%$ from maximum within $100 \mathrm{~cm}$ from soil surface. The comparative area was categorized as Secondary Forest (Latitude 2.938261 ${ }^{\circ}$, Longitude $102.461535^{\circ}$ ). This area was selected to represent non-disturbed soil that had not been subjected to oil cultivation activity. In fact, this area can be considered as riparian reserves or riverine buffer zone, since by law no activity or human interference should take place within 10 meters from a river on the right or left side (Barclay et al. 2017). The downside of this point is that there are no established soil maps to refer. However, the distance is only 471 meters from the test plot, and the structure of the soil was similar to that of the trial site. No other option was available, since all the area has been developed either for oil palm cultivation or housing for people. Urea, rock phosphate, muriate of potash, and kieserite types of fertilizer have been used at field sites of oil palm plantation. The amount of fertilizer used is generally different between immature and mature oil palm trees, with four applications per year. Climatic conditions were in the dry area with rain accumulation of 1500 to $1800 \mathrm{~mm}$ per year. 
Soil samples were collected in January 2014 after running the field experiment planted for 25 years with oil palm. The sampling times were chosen to ensure that there were minimal effects of the most recent fertilizer schedule from the FELDA plantation on the secondary forest at Jalan Kampung Lui (Selatan Serting Hilir, Negeri Sembilan).

\section{Experimental design and soil sampling}

The experiment at each site was designed using a randomized complete block design (RCBD) with four times treatment and four blocks. Soil samples from each of the four plots were collected from 0 to $15 \mathrm{~cm}$ and 15 to $30 \mathrm{~cm}$ depth of soil by taking 16 sub-samples with an auger and subsequently pooling the subsamples from each plot. The treatment plots were labelled as Plot 1 (P1), Plot 2 (P2), Plot 3 (P3), Plot 4 (P4), fertilized area (FA), and unfertilized area (UFA) and with the secondary forest as a control. The soil was spread on a table overnight to dry at room temperature and was sieved through a $5 \mathrm{~mm}$ sieve prior to storage at $-20{ }^{\circ} \mathrm{C}$ in a freezer.

\section{Soil properties and nutrients}

The $\mathrm{pH}$ was determined using a $\mathrm{pH}$ meter (HANNA HI9812). About $20 \mathrm{~g}$ of soil sample was mixed with $50 \mathrm{~mL}$ distilled water and left overnight prior to soil $\mathrm{pH}$ determination (MS, 1980). The total nitrogen and phosphorus contents of the soil samples were determined using a Block-Digester and Auto-Analyser (CNS Analyser, LECO Model - Based on Dumas) according to the SIRIM standard method for soil chemical analysis (MS, 1980). The total organic carbon content of the soil was determined using the Walkely and Black titration method (Gelman et al. 2012). The cation exchange capacity (CEC) was determined by leaching the soil samples with ammonium acetate using the colorimetric method (Mehlich 1980). The analysis of soil exchangeable cation ( $\mathrm{K}, \mathrm{Ca}, \mathrm{Mg}$,) was conducted by leaching the soil samples using 1 $\mathrm{M}$ ammonium acetate $\mathrm{pH} 7$.

\section{Polymerase Chain Reaction (PCR) and Denaturing Gradient Gel Electrophoresis}

Total metagenomic DNA was extracted from $10 \mathrm{~g}$ soil using a MoBio Power soil DNA isolation kit (MoBio Laboraries Inc., Carlsbad, Ca, USA) following the manufacturer's protocol. Total DNA quality and concentration were measured by the Nano Drop ND-2000 spectrophotometric method (Nano Drop Technologies, Thermo Scientific, USA) and by $1 \%$ gel electrophoresis. The PCR was carried out by amplifying the variable region (V3 region) of the $16 \mathrm{~S}$ rDNA using forward and reverse primers. The PCR amplifications were performed in $50 \mu \mathrm{L}$ containing $1.0 \mu \mathrm{L}$ template DNA, 25 $\mu \mathrm{L}$ Ex-Taq DNA polymerase (Takara Shuzo, Japan), $20 \mu \mathrm{L}$ ultrapure water (Millipore), and $2 \mu \mathrm{L}$ of each primer: 357F-with a 40-bp GC clamp (5'-CGC CCG CCG CGC GCG GCG GGC GGG GCG GGG GCA CGG GGG GCC TAC GGG AGG CAG CAG -3 ${ }^{\circ}$ ) and 518R (5'- ATT ACC GCG GCT GCT GG-3'). The PCR amplification was done following the method of Zainudin et al. (2014). The DGGE was performed by loading the PCR product into the well of a 1.5 -mm-thick vertical denaturing $8 \%$ acrylamide gel with a gradient from $30 \%$ to $60 \%$. One hundred percent of the denaturant corresponded to $7 \mathrm{M}$ urea and $40 \%(\mathrm{v} / \mathrm{v})$ formamide. Electrophoresis was performed at $200 \mathrm{~V}$ at 60 ${ }^{\circ} \mathrm{C}$ for $5 \mathrm{~h}$. After electrophoresis, the gel was stained with gelred $(1 \mathrm{mg} / \mathrm{L})$ and viewed with the Gel Doc XR+ System (Biorad laboratories, USA). The denaturing gradient gel electrophoresis (DGGE) band from each lane was excised from the gel with a sterile pipette tip. 


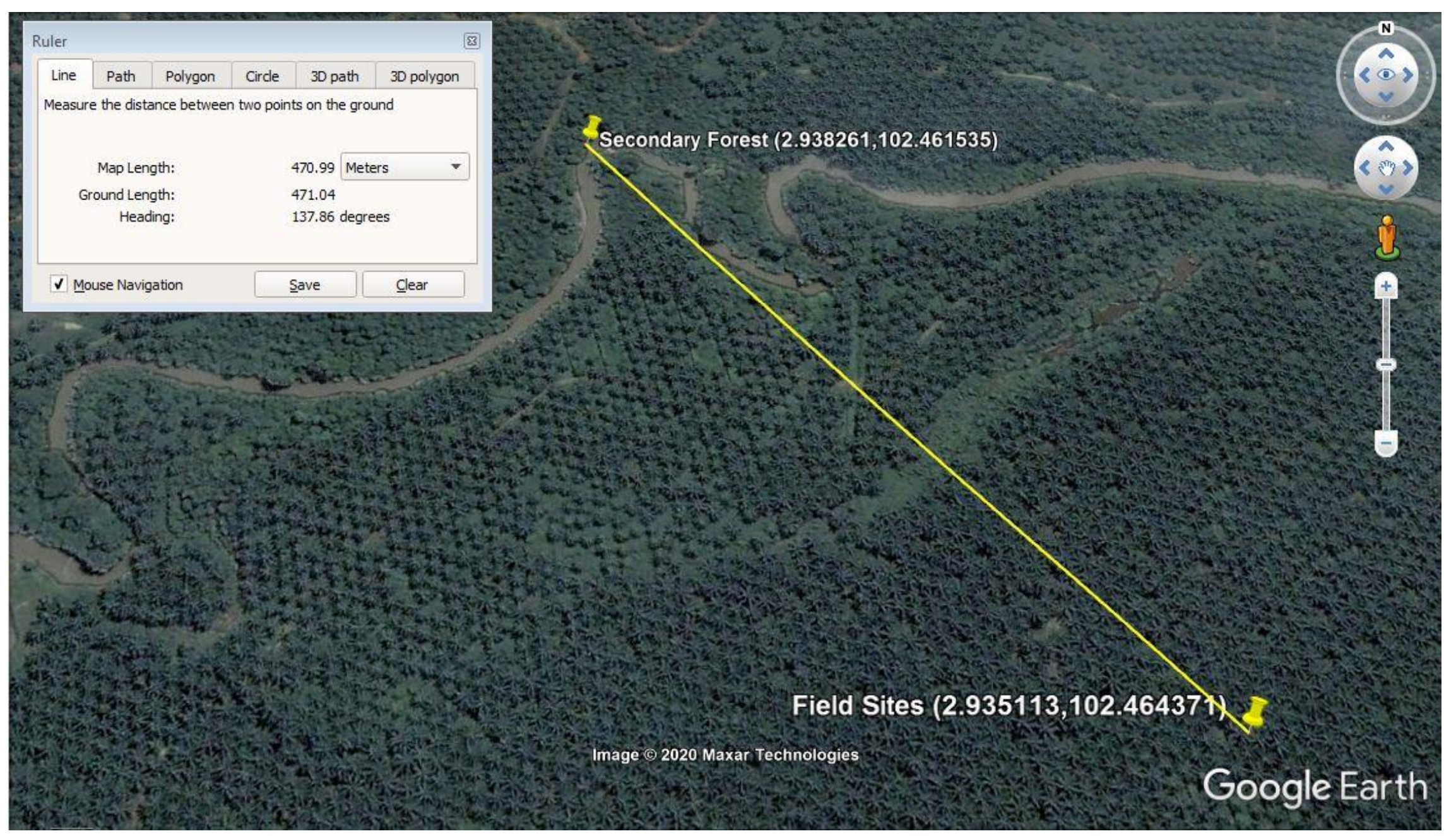

Fig. 1. Visualization of field sites of oil palm plantation and secondary forest using Google Earth 
Each of the excised gel was transferred into a $1.5 \mathrm{~mL}$ tube containing $50 \mu \mathrm{L}$ tris-EDTA buffer ( $\mathrm{pH}$ 8.0). The tube was then incubated overnight to elute the DNA from the gel. The eluted DNA fragment was amplified.

\section{High-throughput $16 S$ rRNA gene sequencing}

The extracted DNA used primers V4-V5 region, forward primer $515 \mathrm{~F}\left(5^{\prime}-\right.$ GTGCCAGCMGCCGCGG-3') and reverse primer 907R (5'CCGTCAATTCMTTTRAGTTT-3'). The $25 \mu \mathrm{L}$ of PCR reaction was comprised of 10x Taq buffer, Taq polymerase (Bio Labs), $20 \mu \mathrm{M}$ of forward and reverse primer, $2 \mathrm{mM}$ dNTP, $25 \mathrm{mM}$ $\mathrm{MgSO}_{4}$ (Toyobo), and $50 \mathrm{ng}$ of cDNA template. The amplification of the DNA sample was done using an initial denaturation temperature at $94{ }^{\circ} \mathrm{C}$ for $3 \mathrm{~min}$, followed by 35 cycles consisting of denaturation at $94{ }^{\circ} \mathrm{C}$ for $45 \mathrm{~s}, 50{ }^{\circ} \mathrm{C}$ for $60 \mathrm{~s}, 72{ }^{\circ} \mathrm{C}$ for $90 \mathrm{~s}$, and a final extension at $72{ }^{\circ} \mathrm{C}$ for $10 \mathrm{~min}$. Following this, the amplicons were purified using Macherey-Nagel, (Duren, Germany). Once the concentrations of the purified PCR products were determined using a Qubit dsDNA HS Assay Kit (Life Technologies, Oregon, (USA), they were processed using Nextera XT DNA library preparation kit according to the Illumina protocol, followed by the sequencing process using the MiSeq sequencing system (Illumina, San Diego, CA). The high-throughput MiSeq data were processed and analysed using QIIME v.1.9.0. (Caporaso et al. 2010). The raw pairedend reads were assembled using the PANDA seq tool, followed by a trimming process to remove low quality and ambiguous reads. The high-quality reads were clustered into operational taxonomic units (OTUs) with $97 \%$ sequence similarity using the de novo OTU picking pipeline. The UCLUST v1.2.22q (DeSantis et al. 2006) software was used to classify each representative sequence prior to aligning the sequences against the Green genes database v13.8 using the PyNAST program (DeSantis et al. 2006; Caporaso et al. 2010). The rarefied OTU tables for the alpha diversity measurement, and rarefaction curves by Shannon diversity metric were used. The beta diversity was analysed by principal coordinate analysis (PCoA) and cJackknife beta-diversity with UPGMA for cluster.

\section{Statistical analyses}

The data for soil properties and nutrient depletion were analyzed by using analysis of variance (ANOVA) with the aid of the SAS software windows version 9.1 (SAS, 2007). Turkey analysis at $p \leq 0.05$ was used to test for significant differences between the treatments. The Tukey's honestly significant difference test for all pairwise comparisons were calculated after ANOVA to compare the treatment means. Statistical analysis of the DGGE profiles using their gel images that were converted, normalized, and digitized using BLAST searches. The neighbour-joining method (Saitou and Nei 1987), evolutionary distance using maximum composite likelihood (Tamura et al. 2004), and evolutionary were analysed in MEGA X (Kumar et al. 2018). QIIME analysis versionV1.9.0, using OUT clustering for alpha and beta diversity (parallel uclust_ref method), and standard UCLUST used de novo OUT at the $97 \%$ similarity level. Alpha diversity estimated with Chaol and ACE indexes, and diversity Shannon. Principal coordinate analysis (PCoA) (Gower 1966) were used to determine the relationship between bacterial taxa within phylum and the sampling area.

\section{RESULTS AND DISCUSSION}

The effects of inorganic fertilizer after 25 years of application on an oil palm plantation were investigated. Table 1 shows that the $\mathrm{pH}$, organic carbon, and cation exchange capacity (CEC) were significantly different between plots at $p<0.05$. 
Samples at plots P1, P2, P3, and P4 with two different depths 0 to $15 \mathrm{~cm}$ and 15 to 30 $\mathrm{cm}$ at the fertilized and unfertilized areas had the same $\mathrm{pH}$ value of 4.5 reduction. However, the $\mathrm{pH}$ was 4.6 at the secondary forest soil, with a reduction of only 0.1 . Previous research at Sarawak physiochemical properties between planted and nonplanted (secondary forest) in oil palm plantation showed the same result with respect to $\mathrm{pH}$ (Hamzah et al. 2009). The value of organic carbon can be reduced by long-term cropping (Pan et al. 2011). Soil organic carbon is the main component in soil organic matter as indicator for soil health (Johns 2017). The CEC and the electrical charge of some of the soil components that contributed to the CEC were affected by the $\mathrm{pH}$ of the soil. The results of the CEC on Table 1 show significant increase when the $\mathrm{pH}$ was decreased. The secondary forest soil value was 3.50 compared with those of P1 to P4 with different depths whose values were about 4.75 until 5.83. The CEC refers to the amount of negative charges available on the surface of the soil particles and indicated the potential of the soil to hold plant nutrients. Therefore, the CEC of the soil was directly affected by the amount and frequency of fertilizer application.

Macronutrient elements included N, P, K, calcium $(\mathrm{Ca})$, magnesium $(\mathrm{Mg})$, and sulphur $(\mathrm{S})$, and micronutrient elements included copper $(\mathrm{Cu})$, iron $(\mathrm{Fe})$, manganese (Mn), and zinc (Zn) (Dhaliwal et al. 2019). Furthermore, the macronutrients after 25 years of inorganic fertilizer being applied showed significant difference at $p \leq 0.05$. Table 3 showed decreases after 25 years' application of inorganic fertilizer from total nitrogen, total phosphorus, and potassium. The total nitrogen content was $0.12 \%$ at the secondary forest, and it decreased between $0.03 \%$ and $0.05 \%$ after 25 -years inorganic fertilizer application. Classification of soil nutrient status for oil palm by Goh (1997) is total nitrogen and organic carbon drop from low in oil secondary forest to very low after 25-years inorganic fertilizer application. Data indicated low total nitrogen because of plant uptake and leaching (Arora and Juo 1982; Cobo et al. 2002). Factors contributing to the losses of organic carbon may include land clearing and subsequent crop cultivation. An important finding regarding nitrogen between secondary forest soil and after 25 years inorganic fertilizer application involved nitrogen stored in organic matter. Soil organic content is prominent in the forest because $\mathrm{N}$ immobilization by trees (Watanabe et al. 2015). Macro and micro-nutrient elements increased after 25years application of inorganic fertilizer such as CEC, available $\mathrm{P}$, calcium, magnesium, manganese, and iron. The CEC is an indicator nutrient adsorption in organo-mineral complexes, in particular preservation by electrostatic basic of cation such as $\mathrm{K}, \mathrm{Mg}, \mathrm{Ca}$ and $\mathrm{Na}$ (Zech et al. 1997). Table 3 shows that the micronutrients boron (B), iron (F), manganese $(\mathrm{Mn})$, zinc $(\mathrm{Z})$, and molybdenum $(\mathrm{Mo})^{+}$contents were significantly different only between plots.

In order to investigate the effects of inorganic fertilizer to the soil structure, the particle size distributions of various samples were studied (Table 4). The results showed that the soil properties (course sand and fine sand) in terms of particle size distributions were significantly different within plots and in the secondary forest soil. Soil structure disruption on cropland was observed compare secondary forest because useful of mechanical machine during land preparation and harvesting process (Watanabe et al. 2015).

The DGGE profiles for the plots after 25 years of inorganic fertilizer applications and forest soil are shown in Fig. 2. Figure 2A shows first DGGE with different sample between fertilizer areas, unfertilized areas, and secondary forest with two different depths with labelled a, b, c, d, e, and f. Figure 2B shows the second DGGE to reconfirm the band from first DGGE. Data clearly showed that sample secondary forest at 0 to $15 \mathrm{~cm}$ labelled as 'e' had eight thick bands (1, 2, 3, 4, 5, 6, 7 and 8). 
Table 1. Soil Properties Composition between Secondary Forest Soil and Soil at Oil Palm Plantation after 25 Years of Inorganic Fertilizer Application in Malaysia

\begin{tabular}{|c|c|c|c|c|c|}
\hline \multirow{2}{*}{ Plot } & \multirow{2}{*}{ Area } & \multirow{2}{*}{$\begin{array}{l}\text { Depth } \\
(\mathrm{cm})\end{array}$} & $\mathrm{pH}$ & Org C & CEC \\
\hline & & & $(1: 2.5)$ & $(\%)$ & $(\%)$ \\
\hline \multirow{4}{*}{$\mathrm{P} 1$} & Fertilizer area & $0-15$ & $4.48 \pm 0.01 \mathrm{~b}$ & $1.11 \pm 0.24 \mathrm{abc}$ & $5.99 \pm 0.40 \mathrm{a}$ \\
\hline & Fertilizer area & $15-30$ & $4.48 \pm 0.05 b$ & $0.87 \pm 0.40 \mathrm{bc}$ & $5.43 \pm 1.04 \mathrm{ab}$ \\
\hline & Unfertilized area & $0-15$ & $4.46 \pm 0.05 b$ & $0.71 \pm 0.17 \mathrm{bc}$ & $5.76 \pm 0.24 a$ \\
\hline & Unfertilized area & $15-30$ & $4.46 \pm 0.05 b$ & $0.92 \pm 0.32 b c$ & $5.91 \pm 0.31 \mathrm{ab}$ \\
\hline \multirow{4}{*}{$\mathrm{P} 2$} & Fertilizer area & $0-15$ & $4.54 \pm 0.01 \mathrm{ab}$ & $0.64 \pm 0.25 c$ & $5.07 \pm 0.66 a$ \\
\hline & Fertilizer area & $15-30$ & $4.54 \pm 0.01 \mathrm{ab}$ & $0.62 \pm 0.21 \mathrm{c}$ & $5.14 \pm 0.50 \mathrm{ab}$ \\
\hline & Unfertilized area & $0-15$ & $4.51 \pm 0.05 \mathrm{~b}$ & $1.15 \pm 0.75 b c$ & $5.61 \pm 0.67 a$ \\
\hline & Unfertilized area & $15-30$ & $4.51 \pm 0.05 b$ & $0.84 \pm 0.42 b c$ & $5.25 \pm 0.65 a b$ \\
\hline \multirow{4}{*}{ P3 } & Fertilizer area & $0-15$ & $4.48 \pm 0.01 \mathrm{~b}$ & $1.13 \pm 0.37 \mathrm{c}$ & $4.80 \pm 0.82 \mathrm{a}$ \\
\hline & Fertilizer area & $15-30$ & $4.48 \pm 0.01 b$ & $0.67 \pm 0.28 c$ & $4.70 \pm 0.28 \mathrm{ab}$ \\
\hline & Unfertilized area & $0-15$ & $4.46 \pm 0.01 \mathrm{~b}$ & $1.19 \pm 0.27 c$ & $5.43 \pm 0.66 \mathrm{a}$ \\
\hline & Unfertilized area & $15-30$ & $4.40 \pm 0.05 b$ & $0.90 \pm 0.35 c$ & $5.34 \pm 0.30 \mathrm{ab}$ \\
\hline \multirow{4}{*}{ P4 } & Fertilizer area & $0-15$ & $4.43 \pm 0.06 \mathrm{~b}$ & $1.07 \pm 0.54 \mathrm{c}$ & $5.14 \pm 0.40 \mathrm{a}$ \\
\hline & Fertilizer area & $15-30$ & $4.41 \pm 0.05 b$ & $0.63 \pm 0.25 c$ & $4.91 \pm 0.46 \mathrm{ab}$ \\
\hline & Unfertilized area & $0-15$ & $4.46 \pm 0.05 b$ & $0.95 \pm 0.05 c$ & $5.71 \pm 0.24 a$ \\
\hline & Unfertilized area & $15-30$ & $4.46 \pm 0.05 b$ & $0.95 \pm 0.39 c$ & $5.31 \pm 0.49 a b$ \\
\hline \multirow{2}{*}{\multicolumn{2}{|c|}{ Soil Forest }} & $0-15$ & $4.70 \pm 0.10 \mathrm{a}$ & $2.30 \pm 0.44 a b$ & $3.57 \pm 0.87 \mathrm{c}$ \\
\hline & & $15-30$ & $4.58 \pm 0.10 \mathrm{ab}$ & $2.74 \pm 0.74 a$ & $4.22 \pm 1.11 \mathrm{c}$ \\
\hline \multicolumn{3}{|c|}{ Treatment } & *** & *** & $* * *$ \\
\hline \multirow{2}{*}{\multicolumn{3}{|c|}{$\begin{array}{cc}\text { Depth } \\
\text { Treatment*Depth }\end{array}$}} & NSD & NSD & NSD \\
\hline & & & NSD & NSD & NSD \\
\hline
\end{tabular}

Salamat et al. (2021). "Soil microbial diversity," BioResources 16(2), 2279-2302. 
Table 2. Soil Macronutrients Composition between Secondary Forest Soil and Soil at Oil Palm Plantation after 25 Years of Inorganic Fertilizer Application in Malaysia

\begin{tabular}{|c|c|c|c|c|c|c|c|c|}
\hline \multirow[b]{2}{*}{ Plot } & \multirow{2}{*}{ Area } & \multirow{2}{*}{$\begin{array}{l}\text { Depth } \\
(\mathrm{cm})\end{array}$} & Total $\mathrm{N}$ & \multicolumn{2}{|c|}{$\mathrm{P}(\mathrm{mg} / \mathrm{kg})$} & $\mathrm{K}$ & $\mathrm{Ca}$ & $\mathrm{Mg}$ \\
\hline & & & $(\%)$ & Total & Avail & \multicolumn{3}{|c|}{ Exch cmol $(+) / \mathrm{kg}$} \\
\hline \multirow{4}{*}{ P1 } & Fertilizer area & $0-15$ & $0.06 \pm 0.03 a b$ & $217.63 \pm 59.90 a$ & $5.72 \pm 0.40 \mathrm{ab}$ & $0.11 \pm 0.01 b$ & $1.12 \pm 0.13 \mathrm{ab}$ & $0.31 \pm 0.08 a$ \\
\hline & Fertilizer area & $15-30$ & $0.07 \pm 0.03 a b$ & $179.38 \pm 21.10 a$ & $5.83 \pm 1.04 a$ & $0.12 \pm 0.04 b$ & $1.00 \pm 0.11 \mathrm{ab}$ & $0.27 \pm 0.07 a$ \\
\hline & Unfertilized area & $0-15$ & $0.06 \pm 0.02 \mathrm{ab}$ & $154.13 \pm 42.80 \mathrm{a}$ & $5.72 \pm 0.24 a b$ & $0.11 b \pm 0.01 b$ & $1.00 \pm 0.18 \mathrm{ab}$ & $0.21 \pm 0.12 a$ \\
\hline & Unfertilized area & $15-30$ & $0.06 \pm 0.02 \mathrm{ab}$ & $189.88 \pm 49.43 a$ & $5.83 \pm 0.31 a$ & $0.12 \pm 0.01 \mathrm{~b}$ & $1.10 \pm 0.18 \mathrm{ab}$ & $0.30 \pm 0.13 a$ \\
\hline \multirow{4}{*}{ P2 } & Fertilizer area & $0-15$ & $0.04 \pm 0.02 \mathrm{ab}$ & $85.13 \pm 43.11 \mathrm{a}$ & $5.72 \pm 0.66 \mathrm{ab}$ & $0.11 \pm 0.04 \mathrm{~b}$ & $1.14 \pm 0.04 \mathrm{ab}$ & $0.33 \pm 0.07 a$ \\
\hline & Fertilizer area & $15-30$ & $0.05 \pm 0.02 \mathrm{ab}$ & $85.13 \pm 41.48 a$ & $5.83 \pm 0.50 a$ & $0.12 \pm 0.02 b$ & $1.10 \pm 0.06 \mathrm{ab}$ & $0.30 \pm 0.06 a$ \\
\hline & Unfertilized area & $0-15$ & $0.04 \pm 0.02 \mathrm{ab}$ & $153.88 \pm 78.37 a$ & $5.72 \pm 067 a b$ & $0.11 \pm 0.03 b$ & $1.22 \pm 0.40 \mathrm{ab}$ & $0.55 \pm 0.34 a$ \\
\hline & Unfertilized area & $15-30$ & $0.04 \pm 0.02 \mathrm{ab}$ & $117.13 \pm 85.95 a$ & $5.83 \pm 0.65 a$ & $0.12 \pm .02 b$ & $1.44 \pm 0.12 b$ & $0.39 \pm 0.11 \mathrm{a}$ \\
\hline \multirow{4}{*}{ P3 } & Fertilizer area & $0-15$ & $0.03 \pm 0.02 b$ & $137.13 \pm 22.84 a$ & $5.72 \pm 0.83 a b$ & $0.11 \pm 0.03 b$ & $1.20 \pm 0.11 \mathrm{ab}$ & $0.40 \pm 0.17 a$ \\
\hline & Fertilizer area & $15-30$ & $0.02 \pm 0.01 \mathrm{~b}$ & $81.38 \pm 49.86 a$ & $5.83 \pm 0.28 a$ & $0.12 \pm 0.05 b$ & $1.00 \pm 0.09 a b$ & $0.27 \pm 0.08 a$ \\
\hline & Unfertilized area & $0-15$ & $0.05 \pm 0.03 a b$ & $128.13 \pm 77.09 a$ & $5.72 \pm 0.66 \mathrm{ab}$ & $0.11 \pm 0.05 b$ & $1.20 \pm 0.22 \mathrm{ab}$ & $0.40 \pm 0.16 a$ \\
\hline & Unfertilized area & $15-30$ & $0.04 \pm 0.02 \mathrm{ab}$ & $123.13 \pm 65.66 \mathrm{a}$ & $5.83 \pm 0.30 a$ & $0.12 \pm 0.04 \mathrm{~b}$ & $1.20 \pm 0.19 \mathrm{ab}$ & $0.32 \pm 0.15 a$ \\
\hline \multirow{4}{*}{ P4 } & Fertilizer area & $0-15$ & $0.03 \pm 0.01 a$ & $141.88 \pm 41.98 a$ & $5.72 \pm 0.40 \mathrm{ab}$ & $0.11 \pm 0.02 b$ & $1.20 \pm 0.20 \mathrm{ab}$ & $0.38 \pm 0.18 a$ \\
\hline & Fertilizer area & $15-30$ & $0.03 \pm 0.01 b$ & $73.13 \pm 13.72 a$ & $5.83 \pm 0.46 a$ & $0.12 \pm 0.02 b$ & $1.00 \pm 0.12 \mathrm{ab}$ & $0.24 \pm 0.11 a$ \\
\hline & Unfertilized area & $0-15$ & $0.06 \pm 0.04 a b$ & $117.88 \pm 37.26 a$ & $5.72 \pm 0.24 a b$ & $0.11 \pm 0.03 b$ & $1.20 \pm 0.17 \mathrm{ab}$ & $0.31 \pm 0.03 a$ \\
\hline & Unfertilized area & $15-30$ & $0.02 \pm 0.02 b$ & $107.13 \pm 42.59 a$ & $5.83 \pm 0.49 a$ & $0.12 \pm 0.03 b$ & $1.11 \pm 0.20 \mathrm{ab}$ & $0.3 \pm 0.19 a$ \\
\hline \multirow{2}{*}{\multicolumn{2}{|c|}{ Soil Forest }} & $0-15$ & $0.12 \pm 0.03 a$ & $136.00 \pm 24.74 a$ & $3.13 \pm 0.50 c$ & $0.28 \pm 0.02 a$ & $1.00 \pm 0.20 \mathrm{ab}$ & $0.23 \pm 0.04 a$ \\
\hline & & $15-30$ & $0.12 \pm 0.03 a$ & $157.75 \pm 60.57 a$ & $3.13 \pm 1.73 c$ & $0.28 \pm 0.02 a$ & $0.62 \pm 0.06 \mathrm{~b}$ & $0.21 \pm 0.04 a$ \\
\hline \multicolumn{3}{|c|}{ Treatment } & $\star * * *$ & NSD & $* \star \star *$ & $* \star * *$ & $* *$ & NSD \\
\hline \multirow{2}{*}{\multicolumn{3}{|c|}{$\begin{array}{c}\text { Depth } \\
\text { Treatment* }{ }^{\star} \text { Depth }\end{array}$}} & NSD & NSD & NSD & NSD & * & NSD \\
\hline & & & NSD & NSD & NSD & NSD & NSD & NSD \\
\hline
\end{tabular}


Table 3. Soil Micronutrients Composition between Secondary Forest Soil and Soil at Oil Palm Plantation After 25 Years Inorganic Fertilizer Application in Malaysia

\begin{tabular}{|c|c|c|c|c|c|c|c|}
\hline Plot & Area & $\begin{array}{l}\text { Depth } \\
\text { (cm) }\end{array}$ & $\begin{array}{c}\mathrm{B} \\
(\mathrm{mg} / \mathrm{kg})\end{array}$ & $\begin{array}{c}\mathrm{Mn} \\
(\mathrm{mg} / \mathrm{kg})\end{array}$ & $\begin{array}{c}\mathrm{Fe} \\
(\mathrm{mg} / \mathrm{kg})\end{array}$ & $\begin{array}{c}\mathrm{Zn} \\
(\mathrm{mg} / \mathrm{kg})\end{array}$ & $\begin{array}{c}\mathrm{Mo} \\
(\mathrm{mg} / \mathrm{L})\end{array}$ \\
\hline \multirow{4}{*}{ P1 } & Fertilizer area & $0-15$ & $1.83 \pm 0.23 a b c$ & $182.28 \pm 11.26 a$ & $953.08 \pm 139.10 a$ & $1.96 \pm 0.07 a b$ & $1.95 \pm 2.41 \mathrm{a}$ \\
\hline & Fertilizer area & $15-30$ & $1.27 \pm 0.40 c$ & $175.92 \pm 13.51 a$ & $789.42 \pm 227.84 a b c$ & $1.93 \pm 0.13 a b$ & $1.31 \pm 2.61 b$ \\
\hline & Unfertilized area & $0-15$ & $1.62 \pm 0.49 a b c$ & $179.25 \pm 7.25 a$ & $814.94 \pm 59.21 \mathrm{abc}$ & $1.92 \pm 0.02 \mathrm{ab}$ & $1.63 \pm 1.04 b$ \\
\hline & Unfertilized area & $15-30$ & $1.67 \pm 0.17 a b c$ & $173.18 \pm 5.29 a$ & $856.63 \pm 105.18 \mathrm{ab}$ & $1.95 \pm 0.05 \mathrm{ab}$ & $2.50 \pm 1.44 \mathrm{~b}$ \\
\hline \multirow{4}{*}{ P2 } & Fertilizer area & $0-15$ & $1.66 \pm 0.18 a b c$ & $195.70 \pm 13.74 a$ & $655.64 \pm 103.21 \mathrm{abc}$ & $1.65 \pm 0.03 a b$ & $1.73 \pm 1.21 b$ \\
\hline & Fertilizer area & $15-30$ & $1.64 \pm 0.13 a b c$ & $199.43 \pm 24.37 a$ & $647.55 \pm 97.12 \mathrm{abc}$ & $1.61 \pm 0.12 b$ & $2.07 \pm 1.44 b$ \\
\hline & Unfertilized area & $0-15$ & $2.00 \pm 0.45 a b c$ & $180.85 \pm 28.11 \mathrm{a}$ & $899.84 \pm 357.52 a$ & $1.76 \pm 0.16 \mathrm{ab}$ & $2.29 \pm 2.00 \mathrm{~b}$ \\
\hline & Unfertilized area & $15-30$ & $1.60 \pm 0.30 \mathrm{bc}$ & $202.17 \pm 31.09 a$ & $615.95 \pm 179.10 \mathrm{abc}$ & $1.59 \pm 0.12 b$ & $0.95 \pm 1.89 b$ \\
\hline \multirow{4}{*}{ P3 } & Fertilizer area & $0-15$ & $1.55 \pm 0.19 \mathrm{bc}$ & $180.91 \pm 8.01 \mathrm{a}$ & $844.73 \pm 144.54 a b$ & $1.79 \pm 0.07 \mathrm{ab}$ & $1.20 \pm 1.97 \mathrm{~b}$ \\
\hline & Fertilizer area & $15-30$ & $1.31 \pm 0.20 \mathrm{~b}$ & $186.79 \pm 31.92 \mathrm{a}$ & $618.47 \pm 124.84 a b c$ & $1.69 \pm 0.08 \mathrm{ab}$ & $2.46 \pm 1.99 \mathrm{~b}$ \\
\hline & Unfertilized area & $0-15$ & $1.61 \pm 0.15 b c$ & $169.63 \pm 45.87 a$ & $821.21 \pm 69.08 \mathrm{abc}$ & $1.76 \pm 0.20 \mathrm{ab}$ & $1.01 \pm 1.26 b$ \\
\hline & Unfertilized area & $15-30$ & $1.45 \pm 0.14 \mathrm{c}$ & $190.98 \pm 37.73 a$ & $725.10 \pm 89.79 a b c$ & $1.75 \pm 0.20 \mathrm{ab}$ & $1.90 \pm 2.11 b$ \\
\hline \multirow{4}{*}{ P4 } & Fertilizer area & $0-15$ & $1.54 \pm 0.31 \mathrm{bc}$ & $185.76 \pm 47.73 a$ & $782.10 \pm 224.38 \mathrm{abc}$ & $1.85 \pm 0.16 \mathrm{ab}$ & $3.18 \pm 2.67 \mathrm{ab}$ \\
\hline & Fertilizer area & $15-30$ & $1.50 \pm 0.30 \mathrm{~b}$ & $166.63 \pm 38.40 \mathrm{ab}$ & $619.47 \pm 116.10 \mathrm{abc}$ & $1.75 \pm 0.06 \mathrm{ab}$ & $3.07 \pm 2.41 b$ \\
\hline & Unfertilized area & $0-15$ & $1.65 \pm 0.17 \mathrm{abc}$ & $185.11 \pm 24.84 a$ & $873.86 \pm 92.60 a$ & $1.82 \pm 0.05 \mathrm{ab}$ & $1.84 \pm 2.21 b$ \\
\hline & Unfertilized area & $15-30$ & $1.22 \pm 0.25 \mathrm{c}$ & $176.90 \pm 22.38 a$ & $797.43 \pm 120.80 \mathrm{abc}$ & $1.79 \pm 0.05 \mathrm{ab}$ & $1.30 \pm 1.53 b$ \\
\hline \multirow{2}{*}{\multicolumn{2}{|c|}{ Soil Forest }} & $0-15$ & $2.43 \pm 0.26 a$ & $70.07 \pm 27.60 \mathrm{bc}$ & $290.75 \pm 70.87 c$ & $1.84 \pm 0.18 \mathrm{ab}$ & $5.02 \pm 0.82 a$ \\
\hline & & $15-30$ & $2.43 \pm 0.24 a$ & $49.02 \pm 22.34 \mathrm{c}$ & $332.08 \pm 55.51 \mathrm{bc}$ & $2.10 \pm 0.26 a$ & $5.83 \pm 1.64 a$ \\
\hline \multicolumn{3}{|c|}{ Treatment } & *** & $\star \star * \star$ & *** & * & ** \\
\hline \multirow{2}{*}{\multicolumn{3}{|c|}{$\frac{\text { Depth }}{\text { Treatment }^{*} \text { Depth }}$}} & ** & NSD & ** & NSD & NSD \\
\hline & & & NSD & NSD & NSD & NSD & NSD \\
\hline
\end{tabular}


Table 4. Soil Particle-Size Distribution between Secondary Forest Soil and Soil at Oil Palm Plantation After 25 Years Inorganic Fertilizer Application in Malaysia

\begin{tabular}{|c|c|c|c|c|c|c|}
\hline \multirow{2}{*}{ Plot } & \multirow{2}{*}{ Area } & \multirow{2}{*}{$\begin{array}{c}\text { Depth } \\
(\mathrm{cm})\end{array}$} & \multicolumn{4}{|c|}{ Particle-Size Distribution } \\
\hline & & & Clay (\%) & Slit (\%) & Coarse sand (\%) & Fine Sand (\%) \\
\hline \multirow{4}{*}{$\mathrm{P} 1$} & Fertilizer area & $0-15$ & $48.42 \pm 0.79 a$ & $36.00 \pm 1.04 a b$ & $11.41 \pm 0.95 a b c$ & $15.05 \pm 1.70 b$ \\
\hline & Fertilizer area & $15-30$ & $50.16 \pm 2.73 a$ & $36.35 \pm 2.21 a$ & $10.59 \pm 3.14 a b$ & $13.78 \pm 1.20 b$ \\
\hline & Unfertilized area & $0-15$ & $48.46 \pm 0.95 a$ & $35.68 \pm 0.70 a b$ & $11.91 \pm 1.70 \mathrm{abc}$ & $14.83 \pm 1.20 \mathrm{~b}$ \\
\hline & Unfertilized area & $15-30$ & $48.38 \pm 0.40 a$ & $35.23 \pm 0.26 \mathrm{abc}$ & $12.04 \pm 1.64 a b c$ & $15.25 \pm 1.37 \mathrm{~b}$ \\
\hline \multirow{4}{*}{$\mathrm{P} 2$} & Fertilizer area & $0-15$ & $48.58 \pm 2.94 a$ & $26.93 \pm 2.06 \mathrm{bcd}$ & $11.61 \pm 2.44 a b c$ & $12.40 \pm 2.11 \mathrm{~b}$ \\
\hline & Fertilizer area & $15-30$ & $47.55 \pm 3.13 a$ & $29.63 \pm 2.73 \mathrm{bcd}$ & $12.74 \pm 2.85 \mathrm{ab}$ & $12.43 \pm 3.63 b$ \\
\hline & Unfertilized area & $0-15$ & $48.73 \pm 2.76 \mathrm{a}$ & $29.33 \pm 1.10 \mathrm{bcd}$ & $12.11 \pm 2.42 \mathrm{abc}$ & $12.08 \pm 1.38 b$ \\
\hline & Unfertilized area & $15-30$ & $47.45 \pm 3.56 a$ & $31.23 \pm 2.91 \mathrm{abcd}$ & $12.04 \pm 2.79 a b c$ & $11.33 \pm 1.44 b$ \\
\hline \multirow{4}{*}{ P3 } & Fertilizer area & $0-15$ & $47.48 \pm 2.61 \mathrm{a}$ & $21.40 \pm 3.06 \mathrm{~cd}$ & $13.34 \pm 4.27 a$ & $11.23 \pm 2.57 \mathrm{~b}$ \\
\hline & Fertilizer area & $15-30$ & $46.45 \pm 3.24 a$ & $21.48 \pm 3.76 \mathrm{~cd}$ & $14.84 \pm 2.77 a$ & $11.40 \pm 1.78 b$ \\
\hline & Unfertilized area & $0-15$ & $45.33 \pm 5.42 a$ & $20.00 \pm 4.86 \mathrm{~d}$ & $14.89 \pm 5.51 \mathrm{a}$ & $13.25 \pm 3.18 b$ \\
\hline & Unfertilized area & $15-30$ & $45.33 \pm 4.19 a$ & $21.20 \pm 5.07 d$ & $13.94 \pm 3.82 \mathrm{a}$ & $12.08 \pm 3.23 b$ \\
\hline \multirow{4}{*}{ P4 } & Fertilizer area & $0-15$ & $46.18 \pm 2.40 a$ & $21.60 \pm 4.32 \mathrm{~cd}$ & $12.29 \pm 3.16 \mathrm{abc}$ & $11.73 \pm 4.10 \mathrm{~b}$ \\
\hline & Fertilizer area & $15-30$ & $48.20 \pm 2.00 a$ & $19.28 \pm 3.79 d$ & $12.29 \pm 2.97 a b c$ & $13.68 \pm 1.98 b$ \\
\hline & Unfertilized area & $0-15$ & $45.80 \pm 2.14 a$ & $20.00 \pm 2.61 d$ & $13.06 \pm 2.97 a$ & $14.28 \pm 0.67 b$ \\
\hline & Unfertilized area & $15-30$ & $45.60 \pm 0.37 a$ & $20.00 \pm 1.36 \mathrm{~d}$ & $14.11 \pm 2.57 \mathrm{a}$ & $10.85 \pm 7.34 b$ \\
\hline \multirow{2}{*}{\multicolumn{2}{|c|}{ Soil Forest }} & $0-15$ & $43.50 \pm 0.91 \mathrm{a}$ & $35.00 \pm 10.02 \mathrm{abc}$ & $2.27 \pm 0.55 \mathrm{c}$ & $28.23 \pm 2.38 \mathrm{a}$ \\
\hline & & $15-30$ & $43.80 \pm 1.39 a$ & $41.02 \pm 6.62 a$ & $2.70 \pm 0.38 \mathrm{bc}$ & $25.70 \pm 1.48 a$ \\
\hline \multicolumn{3}{|c|}{ Treatment } & NSD & 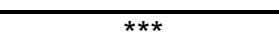 & $\star * * *$ & *** \\
\hline \multirow{2}{*}{\multicolumn{3}{|c|}{$\begin{array}{c}\text { Depth } \\
\end{array}$}} & NSD & NSD & NSD & NSD \\
\hline & & & NSD & NSD & NSD & NSD \\
\hline
\end{tabular}

NSD: Not significantly different statistically not significantly at $p \geq 0.05$; ${ }^{* * *}$ : statistically highly significant at $p \leq 0.001$; **: statistically significant at $p$ $\leq 0.01$; *: statistically significant at $p \leq 0.05$; $(n: 4)$ 
Another sample only had thick bands on number ' 1 ' and ' 2 '. Second DGGE as Fig. 1B reconfirms the appearance of all eight bands. Therefore, the impact of frequent fertilizer applications on soil microbial diversity compared to that of the secondary forest was clearly realized. Soil bacterial diversity differed between each of the depths ( 0 to $15 \mathrm{~cm}$ and 15 to $30 \mathrm{~cm}$ ) of the fertilized and the unfertilized areas in the oil palm plantations, indicating that both treatments affected the microbial diversity in the soil. Detailed analysis of each sample showed that the samples were predominated by different microbial species of each phylum (Fig. 3). The variations of the predominant bacterial species in the secondary forest and also in the oil palm plantation after 25 years of inorganic fertilizer application could be due to the difference in environmental conditions and substrate characteristics, which promoted the growth of certain bacterial species. In this study, the phylogenetic relationships showed that the soils were predominated mainly by Actinobacteria, Firmicutes, and Proteobacteria phyla (Fig. 2.)

Figure 4 shows the relative abundances of the various bacterial communities of the samples. Dominant bacterial phyla at relative abundance $>1 \%$ were Acidobacteria, Proteobacteria, Bacteroidetes, Actinobacteria, Planctomycetes, Firmicutes, and Chloroflexi for all samples. The oil palm plantation soil is mainly dominated in the order by Acidobacteria > Proteobacteria > Actinobacteria > Firmicutes > Bacteroidetes > Chloroflexi whereas the order was Firmicutes > Bacteroidetes > Chloroflexi > Planctomycetes for secondary forest. The results are comparable to the previous studies, which showed that soil in oil palm plantation is dominated mostly by all of these bacterial phyla (Lee-Cruz et al. 2013; Berkelmann et al. 2020; Tang et al. 2020). The results showed that long term continuous cropping decreased the Bacteroides and Firmicutes phyla of the oil palm plantation. In comparison to the secondary forest, the soil after 25 years of inorganic fertilizer applications had higher relative abundances of Acidobacteria, Proteobacteria, and Actinobacteria but lower abundances of Firmicutes. On the other hand, Firmicutes, Bacteroidetes, and Chloroflexi phyla were found to be highly abundant in the secondary forest soil. Previous study by Chaudhari et al. (2020) showed that high abundance of Firmicutes was found in the soils that are rich in organic matter, while the soils with low $\mathrm{pH}$ increase the Acidobacteria abundance. Therefore, higher abundance of Acidobacteria and Chloroflexi over Firmicutes could be attributed to the lower soil organic matter and low $\mathrm{pH}$ of soil after 25 years of inorganic fertilizer as compared to the secondary forest soil (Table 1).

The results also are in agreement to the previous study, which reported that Acidobacteria were highly abundance in oil palm plantation than primary forest soils and their variations were due to the low carbon content and $\mathrm{pH}$ of the soil (Lee-Cruz et al. 2013; Wood et al. 2017). The relative abundance of the top 20 classified bacterial genera showed notable variations between the secondary forest soil and soil after 25 years application of inorganic fertilizer (Fig. 3). Soil physicochemical properties and microbial community structure are mainly affected by different fertilizer management, as indicated by Tang et al. (2020), whereby the application of organic and inorganic fertilizer significantly alter the soil microbial diversity and activities of the paddy field. The three-dimensional principal coordinates analysis (Fig. 4) showed phyla Firmicutes (secondary forest 0 to $15 \mathrm{~cm}$ and 15 to $30 \mathrm{~cm}$ ) and phyla Acidobacteria (fertilizer area 0 to $15 \mathrm{~cm}$ ). Bacterial beta diversity represented using principal coordinate analysis indicated that the bacterial communities were diverse among the samples. Table 5 shows that the Archaea phylum was found to be highly abundant for both samples ( 0 to $15 \mathrm{~cm}$ and 15 to $30 \mathrm{~cm}$ depth levels) of the secondary forest soil. This phylum consisted of genera related to Methanobacterium, Methanobrevibacter, Methanosphaera, Candidatus, Methanolinea, Methanosaeta, Methanomethyloyorans, Methanosarcina, Methanomassiliicocus, and vadin CA11. The dominant bacteria closely related to the 
phylum Acidobacteria were Acidobacterium, Bryocella, Edaphobacteria, Telmatobacter, Terriglobus, Candidatus koribacteria, Geothrix, and Candidatus Solibacter. The dominant bacteria related to the phylum Actinobacteria were Actinotalea, Actinomyces, Actinoalloteichus, Actinokineospora, Arsenicicoccus, Actinomycetales, Actinocatenispora, Actinoplanes, Arthrobacter, Agromyces, Brevibacterium, Cellulomonas, Corynebacterium, Catellatospora, Clavibacter, Cryocola, Curtobacterium, and Candidatus Microthrix.

Figure 6 shows the heat-maps for the secondary forest and the soil after 25 years fertilizer application in the oil palm plantation. The bacterial abundance significantly decreased with increasing years of the oil palm plantation. Fierer et al. (2007) found that soil microbial diversity had almost been completely eradicated after decades of intense agricultural practices in the tall grass prairies of the United States. Similarly, continuous cultivation of cucumber and potatoes caused a reduction in soil bacterial community richness and diversity (Xiang et al. 2014). The soil microbial abundance and diversity play important roles in soil quality, function, and soil ecosystem sustainability. Hence, the loss of soil microbial abundance and diversity might be contributing to the poor growth of plants in continuous cropping systems. During the long years of inorganic fertilizer application to the soil, Firmicutes and Bacteroidetes decreased, whereas Actinobacteria and Proteobacteria were increased. A previous study by Xiang et al. (2014) pointed out that the Bacteroidetes phylum could be a very useful indicator for determining soil health in the vanilla monoculture system and in the culture of black pepper. Moreover, the relative abundance of Firmicutes corresponded with soil-borne disease suppression (Mendes et al. 2011). Phylum Chloroflexus, an indicator for organic carbon, decreased significantly after 25 years application of inorganic fertilizer.

In this study, the microbial community analyses using DGGE and MiSeq were used to observe the side effects of long-term applications of inorganic fertilizer on soil bacterial diversity. As reported earlier, the soil microbial community is essential for maintaining soil health and quality (Garbeva et al. 2006). Microbial activity can be affected by bioavailability of macro and micro-nutrients in soil. In addition, nutrient composition in soil will influence microbial activity. The DGGE profiles showed major variations of microbial community compositions in various types of soil samples. Molecular fingerprinting techniques, including PCR-DGGE analysis, have become popular for assessing diversity, structural composition, and dynamics of microbial communities (Nocker et al. 2007). Although PCR-DGGE allows the rapid assessment of the microbial community's complete structure and the identification of the dominant species, this analytical technique has some limitations. In addition, recovering DNA sequence information from an excised gel band ultimately requires cloning. Only PCR products with relatively small fragment sizes can be separated (up to $500 \mathrm{bp}$ ), and due to the limited success of direct sequencing from an electrophoresed gel band, cloning of amplified 16S rRNA genes is required.

Understanding the correlation between soil properties and microbial diversity is the key to sustainable productivity in oil palm plantations as a continuously cropping system in Malaysia. Application of inorganic fertilizer decreased soil $\mathrm{pH}$ in many studies. In this study, long-term fertilizer application in oil palm plantation only revealed a small decline of $\mathrm{pH}$. The $\mathrm{pH}$ from secondary forest soil was reduced by only 0.1 (from 4.6 to 4.5 ) after 25 years of fertilizer application. Repeated applications of inorganic fertilizer decreased the soil pH (Liu et al. 2012). Long-term applications of inorganic fertilizer might be the factor leading to soil stress and acidification in oil palm plantations. 
Table 5. Percentage of Relative Abundance on Genus of OTU Effect on Treatments

\begin{tabular}{|c|c|c|c|c|c|c|c|c|}
\hline \multirow{2}{*}{ Kingdom } & \multirow{2}{*}{ Phylum } & \multirow{2}{*}{ Related Genus } & \multicolumn{6}{|c|}{ Percentage of relative abundance } \\
\hline & & & FAO & FA30 & UFA0 & UFA30 & SF0 & SF30 \\
\hline Archaea & Euryarchaeota & Methanobacterium & - & - & - & - & 0.099 & 0.369 \\
\hline Archaea & Euryarchaeota & Methanobrevibacter & - & - & - & - & 0.001 & 0.018 \\
\hline Archaea & Euryarchaeota & Methanosphaera & - & - & - & - & - & 0.001 \\
\hline Archaea & Euryarchaeota & CandidatusMethanoregula & - & - & - & - & 0.003 & - \\
\hline Archaea & Euryarchaeota & Methanolinea & - & - & - & - & 0.006 & 0.003 \\
\hline Archaea & Euryarchaeota & Methanospirillum & - & - & - & - & 0.009 & 0.01 \\
\hline Archaea & Euryarchaeota & Methanosaeta & - & - & - & - & 0.164 & 0.096 \\
\hline Archaea & Euryarchaeota & Methanomethylovorans & - & - & - & - & - & 0.006 \\
\hline Archaea & Euryarchaeota & Methanosarcina & - & - & - & - & 0.001 & 0.003 \\
\hline Archaea & Euryarchaeota & Methanomassiliicoccus & - & - & - & - & 0.004 & 0.017 \\
\hline Archaea & Euryarchaeota & vadinCA11 & - & - & - & - & 0.001 & 0.004 \\
\hline Bacteria & Acidobacteria & Acidobacterium & 0.001 & - & 0.003 & - & - & - \\
\hline Bacteria & Acidobacteria & Bryocella & - & - & - & 0.002 & - & - \\
\hline Bacteria & Acidobacteria & Edaphobacter & 0.065 & 0.088 & 0.295 & 0.196 & 0.001 & 0.001 \\
\hline Bacteria & Acidobacteria & Telmatobacter & - & - & 0.006 & - & - & - \\
\hline Bacteria & Acidobacteria & Terriglobus & 0.009 & 0.007 & 0.031 & 0.035 & - & - \\
\hline Bacteria & Acidobacteria & CandidatusKoribacter & 1.223 & 0.703 & 1.507 & 1.935 & 0.007 & 0.003 \\
\hline Bacteria & Acidobacteria & Geothrix & 0.003 & 0.002 & 0.006 & - & - & - \\
\hline Bacteria & Acidobacteria & CandidatusSolibacter & 2.511 & 2.529 & 3.629 & 2.664 & 0.016 & 0.025 \\
\hline Bacteria & Actinobacteria & Actinotalea & 0.012 & 0.005 & 0.009 & 0.004 & 0 & 0.003 \\
\hline Bacteria & Actinobacteria & Actinomyces & 0.103 & 0.035 & 0.057 & 0.028 & 0.001 & - \\
\hline Bacteria & Actinobacteria & Actinoalloteichus & 0.001 & - & - & 0.002 & - & - \\
\hline Bacteria & Actinobacteria & Actinokineospora & 0.001 & - & 0.003 & - & - & - \\
\hline Bacteria & Actinobacteria & Arsenicicoccus & 0.001 & 0.005 & - & 0.002 & - & - \\
\hline Bacteria & Actinobacteria & Actinomycetales & 0.001 & - & - & - & - & - \\
\hline Bacteria & Actinobacteria & Actinocatenispora & - & - & - & 0.002 & - & - \\
\hline Bacteria & Actinobacteria & Actinoplanes & 0.005 & - & 0.006 & - & - & - \\
\hline Bacteria & Actinobacteria & Arthrobacter & 0.247 & 0.342 & 0.279 & 0.081 & - & - \\
\hline Bacteria & Actinobacteria & Agromyces & 0.007 & 0.002 & 0.006 & 0.009 & - & - \\
\hline Bacteria & Actinobacteria & Brevibacterium & 0.004 & - & - & - & - & - \\
\hline Bacteria & Actinobacteria & Cellulomonas & 0.038 & 0.021 & 0.013 & 0.013 & 0.001 & - \\
\hline Bacteria & Actinobacteria & Corynebacterium & 0.007 & - & - & - & - & 0.003 \\
\hline Bacteria & Actinobacteria & Catellatospora & 0.001 & - & - & 0.002 & - & - \\
\hline Bacteria & Actinobacteria & Clavibacter & 0.002 & - & - & - & - & - \\
\hline Bacteria & Actinobacteria & Cryocola & 0.22 & 0.1 & 0.17 & 0.183 & - & - \\
\hline Bacteria & Actinobacteria & Curtobacterium & 0.062 & 0.051 & 0.088 & 0.044 & - & - \\
\hline Bacteria & Actinobacteria & Candidatus Microthrix & 0.001 & - & - & 0.002 & - & - \\
\hline $\begin{array}{l}\text { FA0: Ferti } \\
\text { UFA0: Un } \\
\text { SF0: Secc }\end{array}$ & ndary forest $0-1$ & $\begin{array}{ll}\mathrm{m} & \text { FA30: Fertilizer areas } \\
15 & \text { UFA30: Unfertilized ar } \\
\mathrm{cm} & \text { SF30: Secondary fore } \\
\end{array}$ & $\begin{array}{l}15-30 \mathrm{cr} \\
\text { eas } 15-3 \\
\text { st } 15-30\end{array}$ & $\begin{array}{l}\mathrm{m} \\
30 \mathrm{~cm} \\
\mathrm{~cm}\end{array}$ & & & & \\
\hline
\end{tabular}




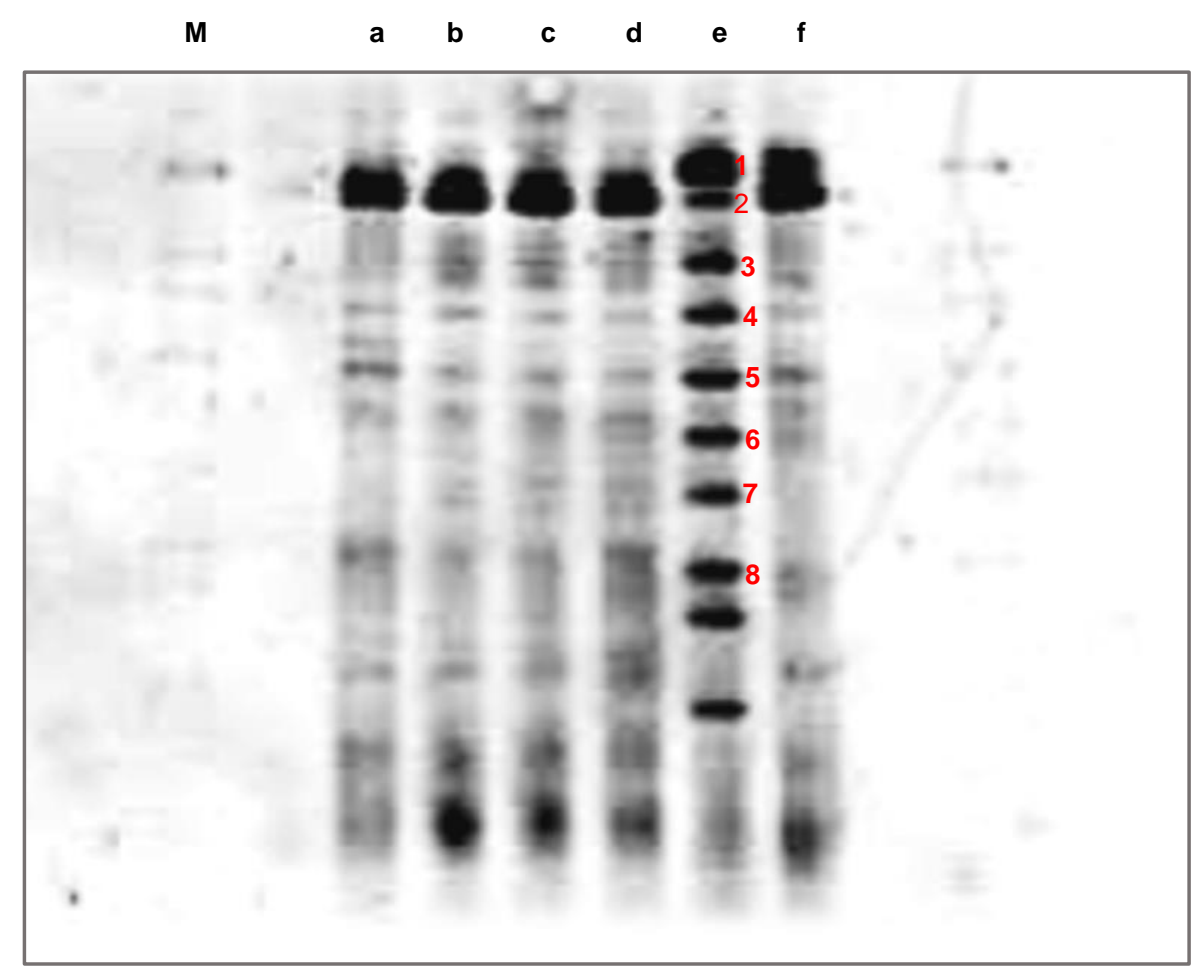

A
M

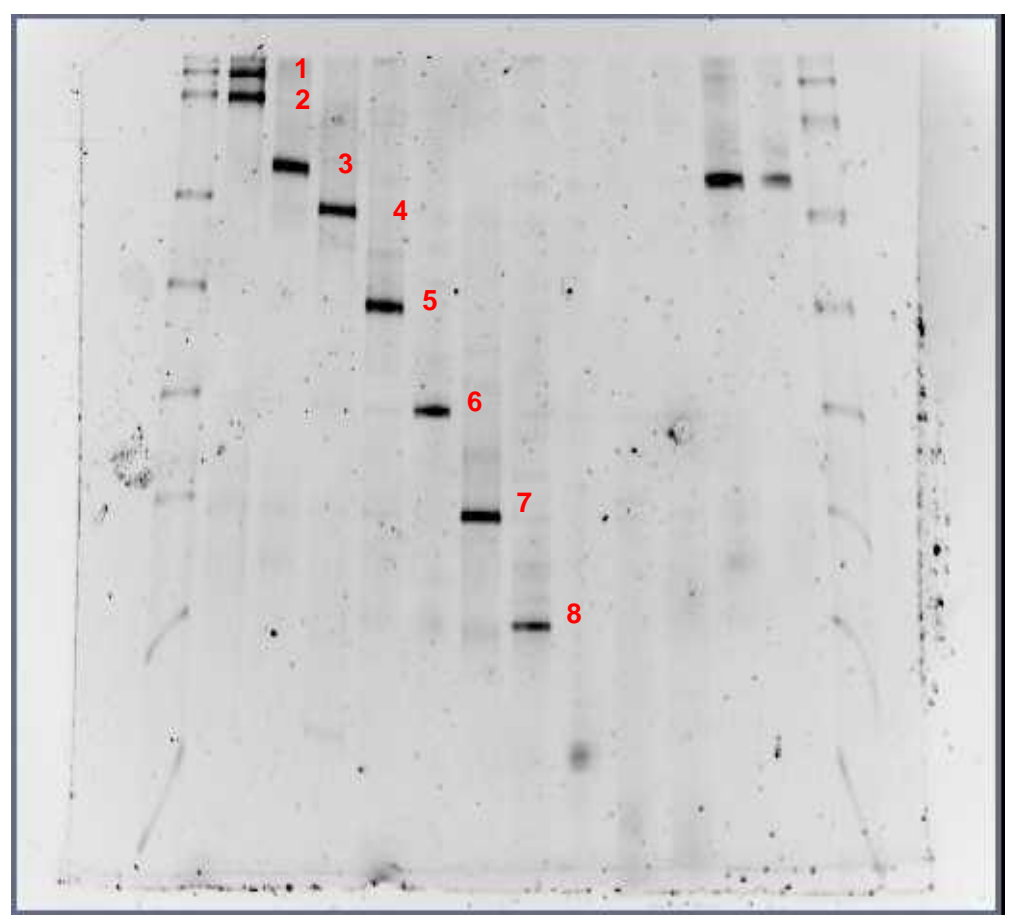

B

Fig. 2A. The DGGE profiles 16S rRNA genes from representative surrounding soils of the secondary forest and of the oil palm plantation after 25 years application of inorganic fertilizer; and Fig. 2B. Secondary DGGE of sample e. The soil samples were from two different depths, $0-5 \mathrm{~cm}$ and $15-30$ $\mathrm{cm}$. The arrow indicates the direction of the electrophoresis and the percentages of the DNA denaturant.

M: Marker; a: Fertilizer areas 0 to $15 \mathrm{~cm}$; b: Fertilizer areas 15 to $30 \mathrm{~cm}$; $\mathbf{c}$ : Unfertilized areas 0 to $15 \mathbf{d}$ : Unfertilized areas $15-30 \mathrm{~cm}$ e: Secondary forest 0 to $15 \mathrm{~cm}$ f: Secondary forest 15 to $30 \mathrm{~cm}$ 


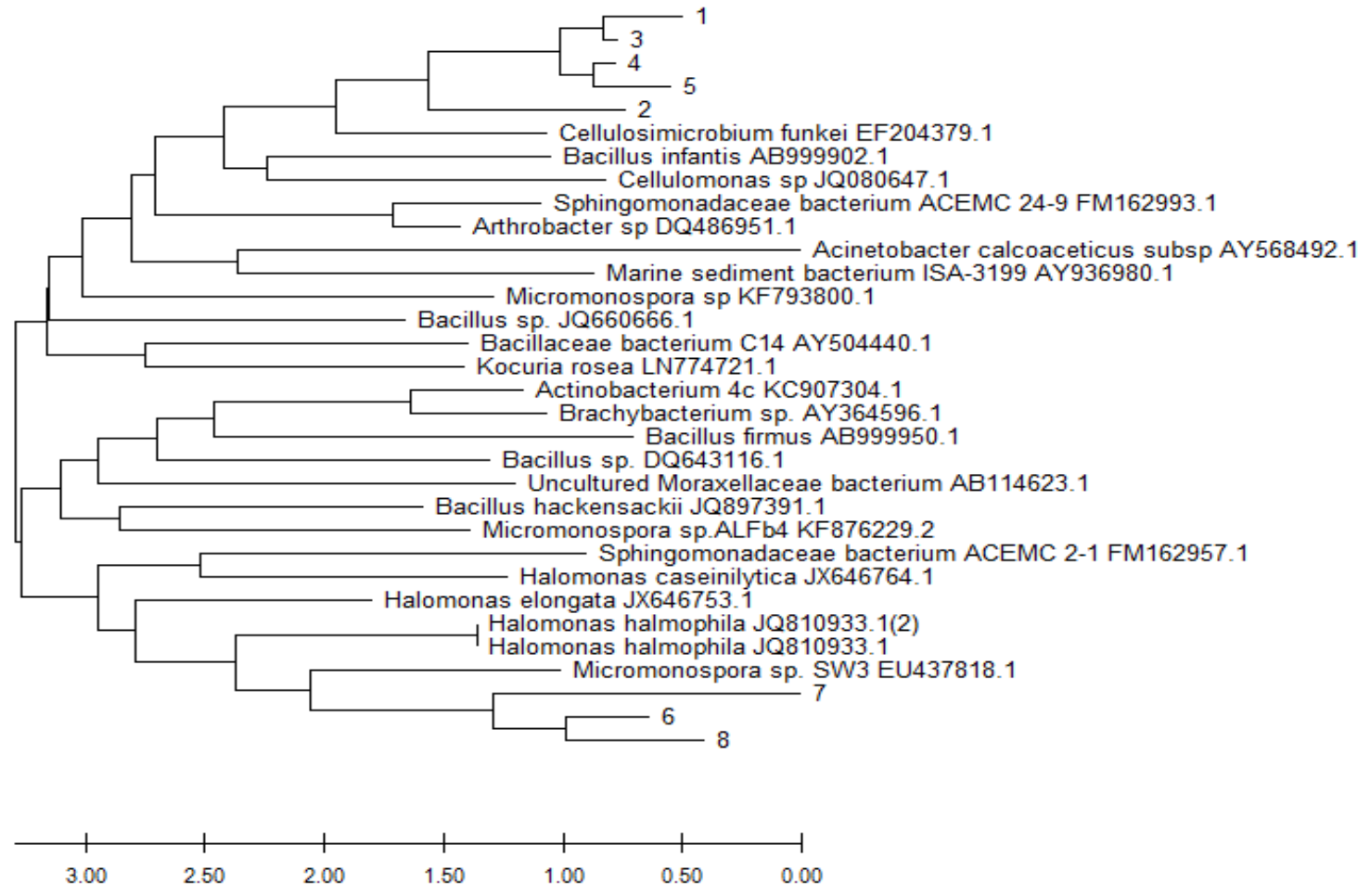

Fig. 3. The evolutionary history was inferred using the Neighbor-Joining method (Saitou and Nei 1987) of the most abundant 16S rDNA sequences originating from secondary forest soil and from oil palm plantation soil after 25 years application of inorganic fertilizer to various closely related sequences obtained from BLAST searches. The optimal tree is shown. The evolutionary distances were computed using the Maximum Composite Likelihood method (Tamura et al. 2004) and are in the units of the number of base substitutions per site. This analysis involved 32 nucleotide sequences. Codon positions included were 1st+2nd+3rd+Noncoding. All ambiguous positions were removed for each sequence pair (pairwise deletion option). There were a total of 1590 positions in the final dataset. Evolutionary analyses were conducted in MEGA X (Kumar et al. 2018) 


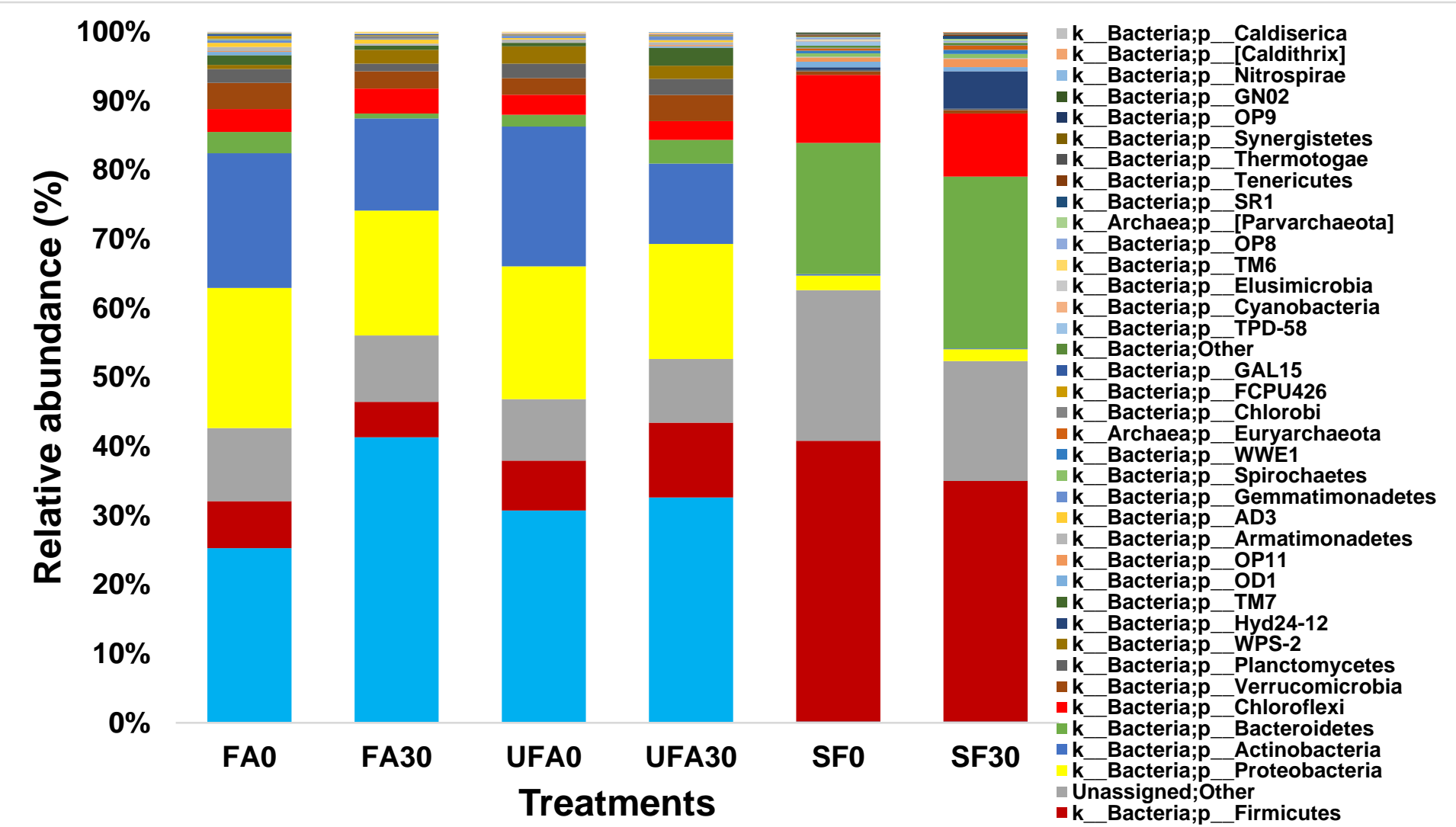

Fig. 4. Distribution of partial sequences of bacterial $16 \mathrm{~S}$ rRNA genes from secondary forest soil and from oil palm plantation soil after 25 years application of inorganic fertilizer at the phylum level with a threshold level of 97.

FA0: Fertilizer areas 0 to $15 \mathrm{~cm}$; FA30: Fertilizer areas 15 to $30 \mathrm{~cm}$; UFA0: Unfertilized areas 0 to 15; UFA30: Unfertilized areas 15 to $30 \mathrm{~cm}$; SF0: Secondary forest 0 to $15 \mathrm{~cm}$; SF30: Secondary forest 15 to $30 \mathrm{~cm}$ 

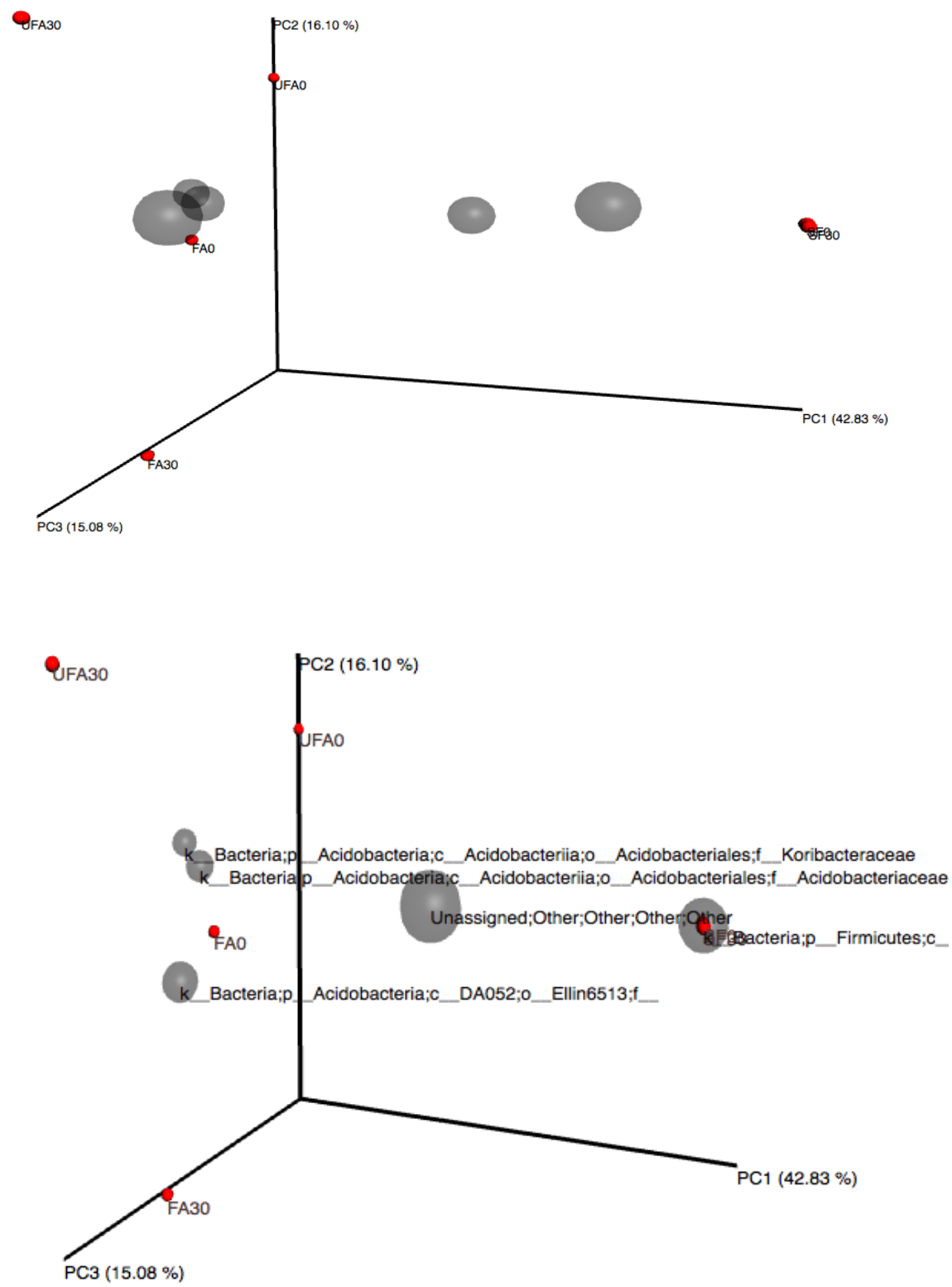

Fig. 5. Three-dimensional principal coordinates analysis plots showing the connection of datasets using the Bray-Curtis method.

FA0: Fertilizer areas 0 to $15 \mathrm{~cm}$; FA30: Fertilizer areas 15 to $30 \mathrm{~cm}$;

UFA0: Unfertilized areas 0 to 15; UFA30: Unfertilized areas 15 to $30 \mathrm{~cm}$

SF0: Secondary forest 0 to $15 \mathrm{~cm}$; SF30: Secondary forest 15 to $30 \mathrm{~cm}$ 


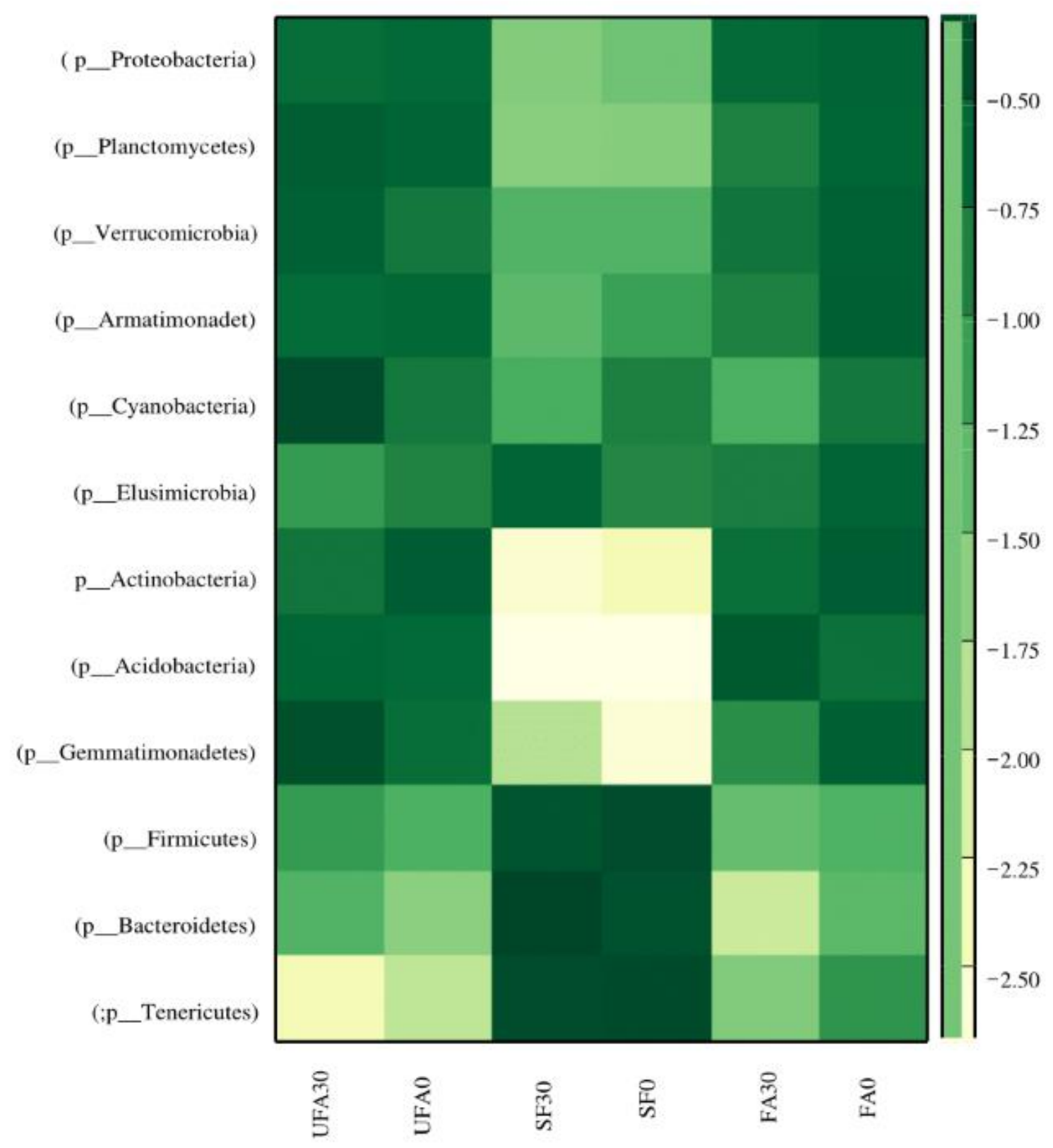

Fig. 6. Heat-map of the secondary forest soil and of oil palm plantation soil after 25 years application of inorganic fertilizer.

FA0: Fertilizer areas 0 to $15 \mathrm{~cm}$

UFA0: Unfertilized areas 0 to $15 \mathrm{~cm}$

SF0: Secondary forest 0 to $15 \mathrm{~cm}$

FA30: Fertilizer areas 15 to $30 \mathrm{~cm}$

UFA30: Unfertilized areas 15 to $30 \mathrm{~cm}$

SF30: Secondary areas 15 to $30 \mathrm{~cm}$ 
Due to the current lack of land space in Malaysia, oil palm is cultivated in acidic soils, to which inorganic fertilizer is added, resulting in leaching and hydrological conditions during rainy seasons (Comte et al. 2013). Classification of soil nutrient status (Goh et al. 1997) showed that CEC at $6 \mathrm{cmol} / \mathrm{kg}$ and total $\mathrm{N}$ content lower than $0.08 \%$ were very prevalent in oil palm plantation. It was demonstrated in this study that when soil acidity increased, more $\mathrm{H}^{+}$ions were attached to the soil and at the same time, the available CEC was decreased. The CEC is an indicator of the potential nutrient adsorption by organic mineral complexes, particularly the retention of basic cations (Zech et al. 1997). From the results previously reported as support data, the continuous cropping often resulted in a decline in soil organic matter (Luo et al. 2015). Long-term application of inorganic fertilizer in oil palm plantation results in macronutrient element contents decreases $(\mathrm{N}$, total $\mathrm{P}$, and $\mathrm{K}$ ) and increases (total available $\mathrm{P}, \mathrm{Ca}$, and $\mathrm{M}$ ). Micronutrient elements such as $\mathrm{B}$, $\mathrm{Mn}, \mathrm{Zn}$, and Mo contents were also decreased, with an increase only in Fe. The results showed that long-term fertilizer application, especially of $\mathrm{N}$, had acidifying effects that causes a decrease in $\mathrm{pH}$ (Fig. 4).

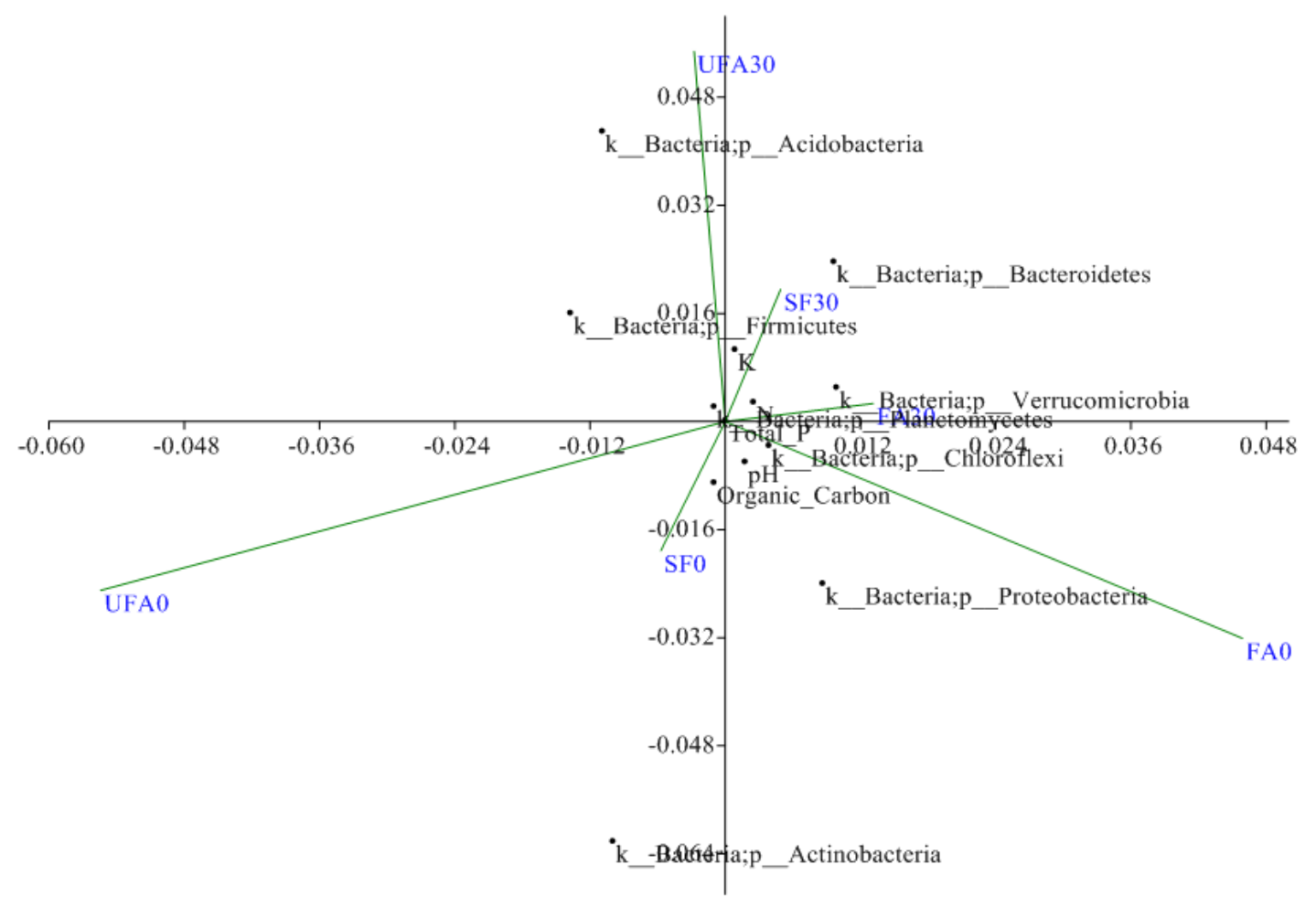

Fig. 7. Principal coordinate analysis of secondary forest soil and soil after 25 years of inorganic fertilizer application.

FA0: Fertilizer areas 0 to $15 \mathrm{~cm}$

UFA0: Unfertilized areas 0 to $15 \mathrm{~cm}$

SF0: Secondary forest 0 to $15 \mathrm{~cm}$

FA30: Fertilizer areas 15 to $30 \mathrm{~cm}$

UFA30: Unfertilized areas 15 to $30 \mathrm{~cm}$

SF30: Secondary areas 15 to $30 \mathrm{~cm}$ 
The Fe content increases were indicators of the acidic soil condition. This confirmed earlier findings that most $\mathrm{N}$ containing fertilizers tended to acidify the soil (Belay et al. 2002). In another study, the high levels of variations observed in the organic content, available $\mathrm{P}, \mathrm{Ca}, \mathrm{Mg}$, and $\mathrm{K}$ could be attributed to the oil palm's impact on the soil, as no fertilization had been carried out on this field for over fifteen years (Ogeh and Osiomwan 2012). Soil acidity can cause distraction in nutrient absorption. The size distribution of soil particles showed big differences between the secondary forest soil and the plantation soil after 25 years application in terms of the percentages of slit, coarse sand, and fine sand. Deceases in the percentage of clay and increases in the percentages of coarse sand, and fine sand were also observed. These observations might be due to the management practices of the oil palm plantation including the usage of machines for fruit harvesting and for cutting grass. Changes in soil properties, macro and micro-nutrient element effect on soil microbial diversity in oil palm plantation. Phyla Firmicutes, Bacteroides and Chloroflexi highly in secondary forest soil switch to Acidobacteria, Proteobacteria, and Actinobacteria in oil palm plantation after long term continuous cropping. Figure 7 shows the principal coordinate analysis of the bacterial treatments. The results showed that the soil organic carbon content was significantly correlated with the secondary forest samples. The samples also positively correlated with the Bacteroidetes phylum. On the other hand, the long-term fertilized soil was significantly correlated with the Firmicutes, Chloroflexi, and Proteobacteria phyla. As described earlier, these communities particularly the Firmicutes play important roles for maintaining soil health.

\section{CONCLUSIONS}

1. Important findings in soil properties as a consequence of long-term continuous cropping included decreases in total nitrogen and organic $\mathrm{C}$ valued from secondary forest. The changes were attributed to land clearing and subsequent plant uptake. The relationship between cation exchange capacity (CEC) and organic $\mathrm{C}$ extent were influenced by $\mathrm{pH}$ of amount and frequency of fertilizer application. The Fe contents was highly elevated, indicating the stressed condition of the soil.

2. Long-term application of inorganic fertilizer in oil palm plantation switch and altered microbial communities in secondary forest. Firmicutes as a suppressor of soil-borne diseases and Bacteroidetes as an indicator of soil health were eliminated in the soil of the oil palm plantation. By contrast, increasing contents of the phyla Acidobacteria, Protobacteria, and Actinobacteria were observed in soil from the oil palm plantation.

\section{ACKNOWLEDGEMENTS}

The authors thank UPM, SATREPS projects (6300156-14201), JASSO, and FELDA for financial supporting. The authors are grateful to the Senior Vice President R\&D / CEO of Felda Agricultural Services Sdn Bhd, Mr. S. Palaniappan for his approval of this joint venture project. The authors would like to thank Mr. Khalifah Khalid, Mr. Zulnaim Dzulkurnain, Mr. Mohd Hafif Samsudin, Mr. Mohammed Abdillah Ahmad Farid, Ms. Norfariza Abd Razak and Ms. Siti Suhaila Sharuddin for their valuable assistance, and Prof. Dr. Tan Soon Guan for the proof reading. 


\section{REFERENCES CITED}

Agnieszka, W., Zofia, S., Aleksandra, B., and Artur, B. (2012). "Evaluation of factors influencing the biomass of soil microorganisms and DNA content," Open Journal of Soil Science 2, 64-69. DOI: 10.4236/ojss.2012.21010

Arora, Y., and Juo, A. S. R. (1982). "Leaching of fertilizer ions in a kaolinitic Ultisol in field plots under cropping and bare fallow," Soil Science Society of American Journal 46, 1212-1218. DOI: 10.2136/sssaj1982.03615995004600060019x

Barclay, H., Gray, C. L., Luke, S. H., Nainar, A., Snaddon, J. L., and Turner, E. C. (2017). RSPO Manual on Best Management Practices (BMPs) for the Management and Rehabilitation of Riparian Reserves, Working Group on 04.04.17. RSPO-GUI T03-003- V1.0 ENG

Belay, A., Claassens, A., and Wehner, F. C. (2002). "Effect of direct nitrogen and potassium and residual phosphorus fertilizers on soil chemical properties, microbial components and maize yield under long-term crop rotation," Biology and Fertility of Soils 35, 420-427. DOI: 10.1007/s00374-002-0489-x

Berkelmann, D., Schneider, D., Hennings, N., Meryandini, A., and Daniel, R. (2020). "Soil bacterial community structures in relation to different oil palm management practices," Scientific Data 7(1), 1-7. DOI: 10.1038/s41597-020-00752-3

Berry, N. J., Phillips, O. L., Lewis, S. L., Hill, J. K., Edwards, D. P., Tawatao, N. B., Ahmad, N., Magintan, D., Khen, C. V., Mohamed, M., Ong, R. C., and Hamer, C. K. (2010). "The high value of logged tropical forests: lessons from northern Borneo," Biodiversity and Conservation 19, 985-997. DOI: 10.1007/s10531-010-9779-z

Chaudhari, D., Rangappa, K., Das, A., Layek, J., Basavaraj, S., Kandpal, B. K., Shouche, Y., and Rahi, P. (2020). "Pea (Pisum sativum L.) plant shapes its rhizosphere microbiome for nutrient uptake and stress amelioration in acidic soils of the northeast region of India," Frontiers in Microbiology 11(June), 1-15. DOI: 10.3389/fmicb.2020.00968

Caporaso, J. G., Bittinger, K., Bushman, F. D., Desantis, T. Z., Andersen, G. L., and Knight, R. (2010). "PyNAST: A flexible tool for aligning sequences to a template alignment," Bioinformatics 26, 266-267. DOI: 10.1093/bioninformatics/btp636

Cobo, J., Barrios, E., Kass, D. C., and Thomas, R. (2002). "Nitrogen mineralization and crop uptake from surface-applied leaves of green manure species on a tropical volcanic-ash soil," Biology and Fertility of Soils 36, 87-92.

DOI: 10.1007/s00374-002-0496-y

Comte, I., Colin, F., Grünberger, O., Follain, S., Whalen, J. K., and Caliman, J.-P. (2013). "Landscape-scale assessment of soil response to long-term organic and mineral fertilizer application in an industrial oil palm plantation, Indonesia," Agriculture, Ecosystems and Environment 169, 58-68. DOI: 10.1016/j.agee.2013.02.010

DeSantis, T. Z., Hugenholtz, P., Larsen, N., Rojas, M., Brodie, E. L., Keller, K., Huber, T., Dalevi, D., Hu, P., and Andersen, G. L. (2006). "Greengenes, a chimera-checked $16 \mathrm{~S}$ rRNA gene database and workbench compatible with ARB," Applied and Environmental Microbiology 72, 5069-5072. DOI: 10.1128/AEM.03006-05

Dhaliwal, S. S., Naresh, R. K., Mandal, A., Singh, R., and Dhaliwal, M. K. (2019). "Dynamics and transformations of micronutrients in agricultural soils as influenced by organic matter build-up: A review," Environmental and Sustainability Indicators 1-2, 1-14. DOI: 10.1016/j.indic.2019.1000007

Dorr de Quadros, P., Zhalnina, K., Davis-Richardson, A., Fagen, J. R., Drew, J., Bayer, 
Camargo, F. A. O., and Triplett, E. W. (2012). "The effect of tillage system and crop rotation on soil microbial diversity and composition in a subtropical acrisol," Diversity 4, 375-395. DOI: 10.3390/d4040375

FAO (2014). FAOSTAT online statistical service. <http://faostat.fao.org/> (accessed 14.03.2020).

Fierer, N., Bradford, M. A., and Jackson, R. B. (2007). "Toward an ecological classification of soil bacteria," Ecology 88, 1354-1364. DOI: 10.1890/05-1839

Fuentes, M., Govaerts, B., De León, F., Hidalgo, C., Dendooven, L., Sayre, K. D., and Etchevers, J. (2009). "Fourteen years of applying zero and conventional tillage, crop rotation and residue management systems and its effect on physical and chemical soil quality," European Journal of Agronomy 30, 228-237. DOI: 10.1016/j.eja.2008.10.005

Gans, J., Woilinsky, M., and Dunbar, J. (2005). “Computational improvements reveal great bacterial diversity and high metal toxicity in soil," Science 309, 13871390. DOI: $10.1126 /$ science. 1112665

Garbeva, P., Postma, J., van Veen, J. A., and van Elsas, J. D. (2006). "Effect of aboveground plant species on soil microbial community structure and its impact on suppression of Rhizoctonia solani AG3," Environmental Microbiology 8, 233-246. DOI: $10.1111 / \mathrm{j} .1462-2920.2005 .00888$

Gower, J. C., (1966). "Some distance properties of latent root and vector methods used in multivariate analysis," Biometrika 53, 325-338. DOI: 10.1093/bio-met/53.3-4.325

Gelman, F., Binstock, R., and Halicz, L. (2012). "Application of the Walkley-Black titration for the organic carbon quantification in organic rich sedimentary rocks," Fuel 96, 608-610. DOI: 10.1016/j.fuel.2011.12.053

Goh, K. J. (1997). "Fertilizer recommendation systems for oil palm: Estimating the fertilizer rates," pp. 1-37. http://www.smart-fertilizer.com/articles/Cation-ExchangeCapacity.

Gunarso, P., Hartoyo, M. E., Agus, F., and Killen, T. J. (2013). "Oil palm and land use change in Indonesia, Malaysia and Papua New Guinea," Reports from the Technical Panels of $2^{\text {nd }}$ Greenhouse Gas Working Group of the Roundtable on Sustainable Palm Oil (RSPO), pp. 29-63.

Huang, L-F., Song, L-X., Xia, X-J., Mao, W-H., Shi, K., Zhou, Y-H., and Yu, J-Q. (2013). "Plant-soil feedbacks and soil sickness: from mechanisms to application in agriculture," Journal of Chemical Ecology 39, 232-242. DOI: 10.1007/s10886-0130244-9

Johns, C. (2017). "The role of carbon in promoting healthy soils," Future Directions International 2017, 1-14

Kumar, S., Stecher, G., Li, M., Knyaz, C., and Tamura, K. (2018). "MEGA X: Molecular evolutionary genetics analysis across computing platforms," Molecular Biology and Evolution 35, 1547-1549. DOI: 10.1093/molbev/msy096

Ogeh, J. S., and Osiomwan, G. E. (2012). "Evaluation of the effect of oil palm on some physical and chemical properties of rhodic paleudults," Nigerian Journal of Basic and Applied Science 20, 78-82

Lee-Cruz, L., Edwards, D. P., Tripathi, B. M., and Adams, J. M. (2013). "Impact of logging and forest conversion to oil palm plantations on soil bacterial communities in borneo," Applied and Environmental Microbiology, 79(23), 7290-7297. DOI: 10.1128/AEM.02541-13 
Liu, Y., Shi, G., Mao, L., Cheng, G., Jiang, S., Ma, X., An, L., Du, L., Johnson, N. C., and Feng, H. (2012). "Direct and indirect influences of 8 year of nitrogen and phosphorus fertilization on Glomeromycota in an alpine meadow ecosystem," New Phytologist 194, 523-535. DOI: 10.1111/j.1469-8137.2012.04050.x

Liu, X., Zhang, J., Gu, T., Zhang, W., Shen, Q., Yin, S., and Qiu, H. (2014). "Microbial community diversities and taxa abundances in soils along a seven-year gradient of potato monoculture using high throughput pyrosequencing approach," PLoS One 9, 110. DOI: 10.1371/journal.pone.0086610

Luo, P., Han. X., Wang, Y., Han, M., and Shi, H. (2015). "Influence of long-term fertilization on soil microbial biomass, dehydrogenase activity, and bacterial and fungal community structure in a brown soil of northeast China," Ann. Microbiol. 65, 533-542. DOI: 10.1007/s13213-014-0889-9

Mehlich, A. (1980). "The operation of soil and plant testing services in the USA," FAO Soils Bulletin 38, 37-45

Mendes, R., Kruijt, M., de Bruijn, I., Dekkers, E., van der Voort, M., Schneider, J. H. M., Piceno, Y. M., DeSantis, T. Z., Andrsen, G. L., Bakker, P. A. H. M., and Raaijimakers, J. M. (2011). "Deciphering the rhizosphere microbiome for diseasessuppressive bacteria," Science 332, 1097-1100. DOI: 10.1126/science.1203980

Malaysian Standard MS 678 (1980). "Recommended methods for soil chemical analysis: Part IV: MS 678," Standards and Industrial Research Institute of Malaysia, Shah Alam.

Nocker, A., Lepo, J. E., Martin, L. L., and Snyder, R. A. (2007). "Response of estuarine biofilm microbial community development to changes in dissolved oxygen and nutrient concentrations," Microbial Ecology 54, 532-542. DOI: 10.1007/s00248-0079236-Z

Pan, Y., Birdsey, R. A., Fang, J., Houghton, R., Kauppi, P. E., Kurz, W. A., Phillips, O. L., Shvidenko, A., Lewis, S. L., Canadell, J. G., Ciais, P., Jackson, R. B., Pacala, S. W., McGuire, A. D., Piao, A. R., Sitch, S., and Hayes, D. (2011). "A large and persistent carbon sink in the world's forests," Science 333, 988-993. DOI: 10.1126/science. 1201609

Paramananthan, S. (2020). "Chapter 15, Ultisols," in: Malaysian Soil Taxonomy, S. Paramananthan (ed.), Revised Third Edition, CAB International, pp. 125-162.

Roesch, L. F. W., Fulthorpe, R. R., Riva, A., Casella, G., Hadwin, A. K. M., Kent, A. D., Kent, A. D., Daroub, S. H., Camargo, F. A. O., Farmerie, W. G., and Triplett, E. W. (2007). "Pyrosequencing enumerates and contrasts soil microbial diversity," The ISME Journal 1, 283-290. DOI: 10.1038/ismej.2007.53

Saitou, N., and Nei, M. (1987). "The neighbor-joining method: A new method for reconstructing phylogenetic trees," Molecular Biology and Evolution 4, 406-425.

Statistical Analysis Systems Institute (2007). Statistical Analysis Systems Computer Package, SAS Institute, Cary, N. C.

Tang, H., Li, C., Xiao, X., Shi, L., Cheng, K., Wen, L., and Li, W. (2020). "Effects of short-term manure nitrogen input on soil microbial community structure and diversity in a double-cropping paddy field of southern China," Scientific Reports, Nature Publishing Group UK, 10, 1-9. DOI: 10.1038/s41598-020-70612-y

Tamura, K., Nei, M., and Kumar, S. (2004). "Prospects for inferring very large phylogenies by using the neighbor-joining method," Proceedings of the National Academy of Sciences (USA) 30, 11030-11035. DOI: 10.1073/pnas.0404206101 
Watanabe, Y., Kikuno, H., Asiedu. R., Masunaga, T., and Wakatsuki T. (2015).

"Comparison of physicochemical properties of soil under constrasting land use systems in Southwestern Nigeria," Japan Agriculture Research Quarterly 49, 319331. DOI: $10.6090 /$ jarq.49.319

Wicke, B., Sikkema, R., Dornburg, V., and Faaij, A. (2011). "Exploring land use changes and the role of palm oil production in Indonesia and Malaysia," Land Use Policy 28, 193-206. DOI: 10.1016/j.landusepol.2010.06.001

Wood, S. A., Gilbert, J. A., Leff, J. W., Fierer, N., D’Angelo, H., Bateman, C., Gedallovich, S. M., Gillikin, C. M., Gradoville, M. R., Mansor, P., Massmann, A., Yang, N., Turner, B. L., Brearley, F. Q., and McGuire, K. L. (2017). "Consequences of tropical forest conversion to oil palm on soil bacterial community and network structure," Soil Biology and Biochemistry 112, 258-268. DOI: 10.1016/j.soilbio.2017.05.019

Xiang, W., Zhao, Q., Zhao, J., Xun, W., Li, R., Zhang, R., Wu, H., and Shen, Q. (2014). "Different continuous cropping spans significantly affect microbial community membership and structure in a vanilla-grown soil as revealed by deep pyrosequencing," Soil Microbiology 70, 209-218. DOI: 10.1007/s00248-014-0516-0

Yang, J. I., Ruegger, P. M., McKenry, M., V, Becker, J. O., and Borneman, J. (2012). "Correlations between root-associated microorganisms and peach replant disease symptoms in a California soil," PLoS One 7, 1-10. DOI: 10.1371/journal.pone.0046420

Zainudin, M. H. M., Hassan, M. A., Abdullah, N., Md Shah, U. K., Tokura, M., Yasueda, H., Sakai, K. and Shirai,Y. (2014). "Bacterial community and biochemical changes during composting of lignocellulose oil palm empty fruit bunch," BioResources 9(1), 316-335. DOI: 10.15376/biores.9.1.316-335

Zech, W., Senesi, N., Guggenberger, G., Kaiser, K., Lehmann, J., Miano, T. M., and Schroth, G. (1997). "Factors controlling humification and mineralization of soil organic matter in the tropics," Geoderma 79, 117-161. DOI: $10.1016 / \mathrm{S} 0016(97) 00040-2$

Article submitted: September 9, 2020; Peer review completed: January 9, 2021; Revised version received and accepted: January 28, 2021; Published: February 2, 2021.

DOI: 10.15376/biores.16.2.2279-2302 